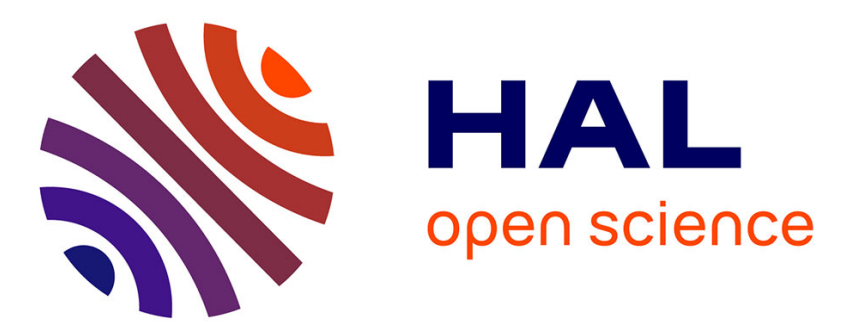

\title{
Magnetic responsive polymer composite materials
}

Julie Thevenot, Hugo de Oliveira, Olivier Sandre, Sébastien Lecommandoux

\section{To cite this version:}

Julie Thevenot, Hugo de Oliveira, Olivier Sandre, Sébastien Lecommandoux. Magnetic responsive polymer composite materials. Chemical Society Reviews, 2013, 42 (17), pp.7099-7116. $10.1039 / \mathrm{c} 3 \mathrm{cs} 60058 \mathrm{k}$. hal-00926554

\section{HAL Id: hal-00926554 https://hal.science/hal-00926554}

Submitted on 14 Dec 2018

HAL is a multi-disciplinary open access archive for the deposit and dissemination of scientific research documents, whether they are published or not. The documents may come from teaching and research institutions in France or abroad, or from public or private research centers.
L'archive ouverte pluridisciplinaire HAL, est destinée au dépôt et à la diffusion de documents scientifiques de niveau recherche, publiés ou non, émanant des établissements d'enseignement et de recherche français ou étrangers, des laboratoires publics ou privés. 


\title{
Magnetic Responsive Polymer Composite Materials
}

\author{
Julie Thévenot ${ }^{a}$, Hugo Oliveira ${ }^{a}$, Olivier Sandre ${ }^{* a}$, and Sébastien Lecommandoux ${ }^{* a}$ \\ Received 10th February 2013, Accepted 2nd May 2013 \\ DOI: $10.1039 / \mathrm{c3} \operatorname{cs60058k}$
}

\begin{abstract}
Magnetic responsive materials are the topic of intense research due to their potential breakthrough applications in the biomedical, coatings, microfluidics and microelectronics fields. By merging magnetic and polymer materials one can obtain composites with exceptional magnetic responsive features. Magnetic actuation provides unique capabilities as it can be spatially and temporally controlled, and additionally be operated externally to the system, providing a non-invasive approach to remote control. We identified three classes of magnetic responsive composite materials, according to their activation mode and intended applications, which can be defined by the following aspects. A) Their ability to be deformed (stretching, bending, rotation) upon exposure to magnetic field. B) The possibility to remotely drag them to a targeted area, called magnetic guidance, which is particularly interesting for biomedical applications, including cell and biomolecule guidance and separation. C) The opportunity to use magnetic induction for thermoresponsive polymer materials actuation, which has shown promising results for controlled drug release and shape memory devices. For each category, essential design parameters that allow a fine-tuning of the properties of these magnetic responsive composites are presented using key examples.
\end{abstract}

\section{Key Learning Points box.}

In this tutorial review, a critical overview is proposed to the reader, providing fundamental knowledge about:

1. How to obtain efficient magnetic-responsive polymer-based materials and what are the critical issues met while designing them?

2. What are the main application fields for magnetic responsive composite polymer materials?

3. Which type of response can be elicited in a polymer material through magnetic stimulus?

4. What are the physical mechanisms involved in the transduction of the magnetic trigger into the desired response?

5. What are the usual characteristics (homogeneous/gradient, permanent/alternating, amplitude) of the magnetic fields employed for each type of application?

\section{Introduction}

Stimuli-responsive materials have drawn a lot of interest over the last decades due to their biomimetic behaviour and their potential use in smart or intelligent devices. Indeed, in any living systems, adaptation and ability to respond to environmental changes from the molecular to macromolecular level is fascinating and crucial for maintaining and regulating normal functions. Stimuliresponsive or smart materials are then often defined as materials that can respond to $\mathrm{pH}$, light, temperature, ionic strength, electric or magnetic field variations - for the most commonly used triggers - by changing their own properties: changes in size, surface area, solubility, permeability, shape, mechanical, optical properties, among others.

In this research area, polymer-based materials are the most developed and studied, owing to their high versatility and ability to dramatically alter their intrinsic properties as a function of small environmental changes. ${ }^{1}$ Smart polymers are increasingly playing an important role in a wide range of applications, especially in the biomedical field (drug delivery, tissue engineering, biosensors, active diagnosis), coatings (smart textiles and fibres) and microelectronics (actuators, electromechanics). Indeed, the huge development of synthesis and successes in polymer chemistry allow a proper design of well-defined macromolecules that can incorporate stimuliresponsive building blocks for all the above-mentioned triggers. However, intrinsically magnetic responsive polymers are scarce and generally present a poor efficacy. Liquid-crystalline polymers and elastomers have been designed for application as artificial muscle, based on the seminal ideas proposed by P.-G. de Gennes. ${ }^{2}$ Combined effects of magnetic orientation, and temperature induced phase transition (from nematic to isotropic phase) are at the origin of volume changes and deformations. In spite of their interesting mechanical and thermal properties, liquid crystal polymers require intense magnetic fields for their alignment $\left(H \sim 10^{3} \mathrm{kA} / \mathrm{m}\right)$ and present low switching rates. Indeed, their response time is limited by their high viscosity, especially in the bulk, and high temperatures are often required to reach the appropriate phase transition. ${ }^{3}$ Contrary to systems limited by diffusion processes (of either heat or mass), those directly operated by exposure to a field (be it magnetic or electric) might have shorter response times, possibly as fast as natural skeletal muscles. ${ }^{4}$

To produce highly efficient magnetic-responsive materials, the "doping" of polymer materials with magnetic nanoparticles (MNPs), made of inorganic matter (most often superparamagnetic iron oxide $\mathrm{Fe}_{3} \mathrm{O}_{4}$ or $\gamma-\mathrm{Fe}_{2} \mathrm{O}_{3}$, or "soft" metallic iron, but also "hard" magnetic materials e.g. $\mathrm{Co}, \mathrm{Ni}$, $\mathrm{FeN}, \mathrm{FePt}, \mathrm{FePd} . .$.$) appeared to be the more appealing and$ efficient solution. Indeed, the magnetic moment of these "small" 
magnets, much larger than those of molecular magnets, allows them to respond to weak stimuli (static or alternating magnetic field) with a significant effect (e.g. movement, heat generation, magnetic or optical signal). The resulting composites that can be named "magnetic responsive polymer composites" (MRPCs) are the topic of this review. The focus is thus different from the 2009 review by C. S. Brazel ${ }^{5}$ on "magnetothermally-responsive nanomaterials" dedicated mostly to thermo-sensitive polymers associated to MNPs, a class of MRPCs that will also be discussed here. Our approach is also different from Dai and Nelson's in their 2010 tutorial review on magnetic-responsive polymer composites, ${ }^{6}$ and from the review by Medeiros et al on stimuliresponsive magnetic particles for biomedical applications which sensitivity to a magnetic field was not always coupled to the other stimuli $(\mathrm{pH}$, light, temperature, electric field, ionic strength...). ${ }^{7}$ In addition to the mention of the latest works on the subject, we opted indeed for a totally different approach, based on applications rather than preparation methods. Under our focus, the activation mode of MRPCs always consists in the application of a magnetic field, either static $(H d c)$ or alternating ( $H a c$ ). In our view of MRPCs, magnetism must determine their core responses rather than be an additional functionality.
Magnetic nanoparticles, when combined with a polymer material having its own properties, can produce a large variety of MRPCs. Hence, the main core of this review will be organized into three parts (Figure 1), depending on the merged properties resulting from the composites. When magnetic nanoparticles are incorporated into elastomeric polymer scaffolds, controlled deformations can be obtained such as stretching or contraction of a cylinder, bending of an elongated sample, deflection of a membrane, chaining of microparticles, rotation of anisotropic objects, rupture of a capsule (section 2, Figure 1 A, B and C). Composites made from biocompatible polymer matrices have been recently studied for magnetic guidance and separation, such as magnetic cell targeting and manipulation, and for sorting and separation applications: to this aim, a magnetic field gradient is applied at a selected location in a flow circulation such as in the blood circulation or a fluidic device (section 3, Figure 1 D, E and F). Combined with thermosensitive polymers, the activation of magnetic nanoparticles, when exposed to an alternating magnetic field, can create localized heating that was successfully used for both controlled drug release and shape memory actuators (section 4, Figure $1 \mathrm{G}$ and $\mathrm{H}$ ).

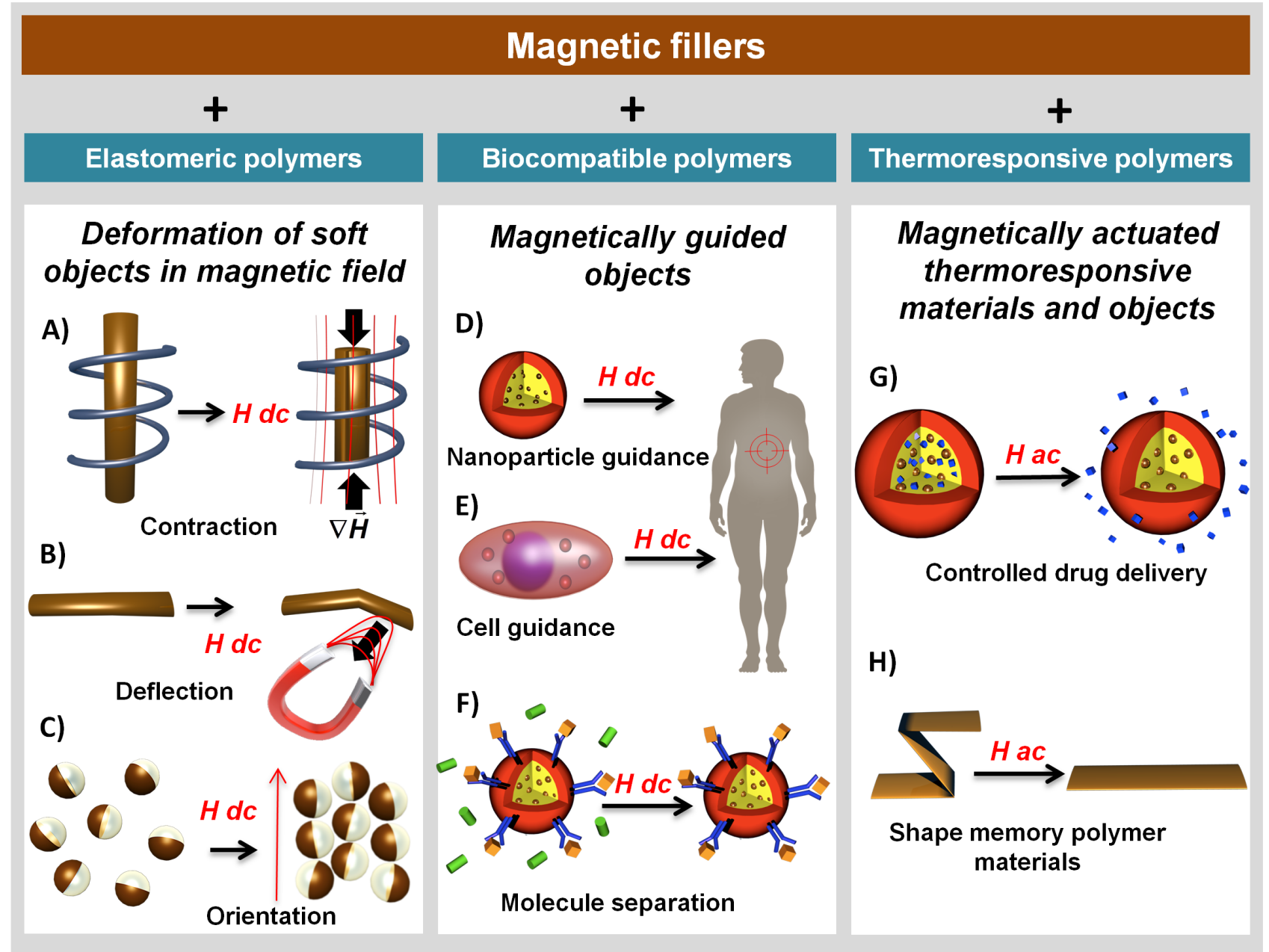

Fig. 1 Schemes depicting the different types of magnetic responsive materials obtained from the doping of various polymers with magnetic particles and illustration of their response when exposed to a static magnetic field $(H d c)$ or to an alternating magnetic field $(H a c)$. From left to right: composites made from elastomeric polymers can be deformed in homogeneous fields or gradients in a controlled fashion; MRPC particles made of polymers designed for biomedical applications can be used for magnetic guidance for drug delivery or separation purpose; MRPC from thermoresponsive polymers can be activated by magnetic induction using alternating fields. 


\section{Deformation of soft materials in magnetic fields}

This section deals with magnetic composite polymer materials that can be referred to as "soft matter" for their mechanical response (low elastic modulus) and "soft magnet" for their magnetic behaviour (with no remanent magnetization in absence of an applied field). The first reported materials of this class were obtained by association of a ferrofluid and a hydrogel, thus named "ferrogels".

\subsection{Ferrogels: macroscopic deformation in field gradients}

In their pioneering work, M. Zrinyi, L. Barsi and A. Büki loaded an aqueous dispersion of magnetite $\left(\mathrm{Fe}_{3} \mathrm{O}_{4}\right)$ nanoparticles - with a diameter around $10 \mathrm{~nm}$ - within a glutaraldehyde cross-linked poly(vinyl alcohol) (PVA) hydrogel. Shaped as cylinders, these ferrogels exhibited stretching, contraction or bending deformations when exposed to an inhomogeneous static magnetic field (Figure 2). ${ }^{8}$

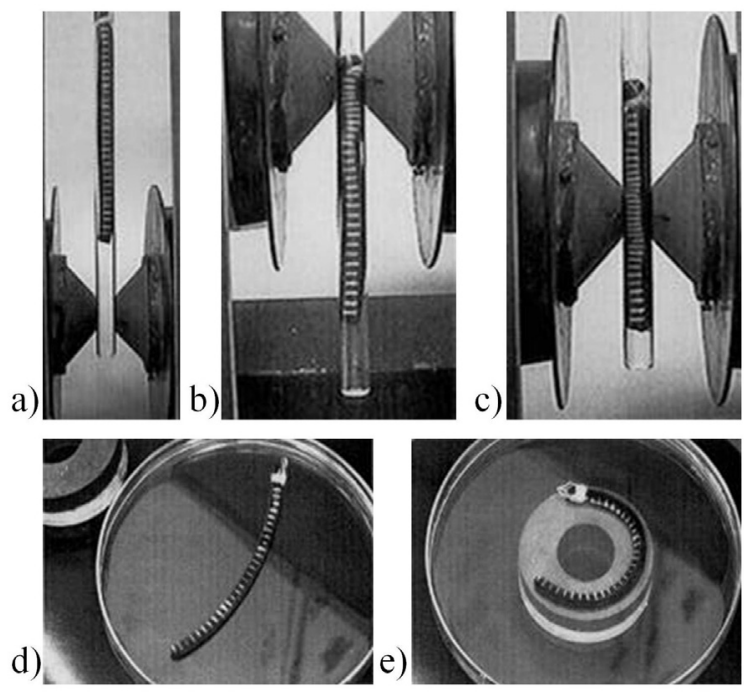

Fig. 2 Elongation, contraction or bending of a ferrogel in non uniform magnetic field created by the polar pieces guiding the field lines of an electromagnet: a) no magnetic field applied; b) the maximal field strength is located on the top surface of the ferrogel; c) the maximal field strength is focused in the middle of the gel along its axis; d) the gel is located $15 \mathrm{~cm}$ from the magnet; e) bending on a toroidal permanent magnet (adapted from ref. ${ }^{8}$ with permission. Copyright 1996, American Institute of Physics. Pictures from the original publication available on the authors' website).

Since then, many other hydrophilic polymers were proposed to host magnetic nanoparticles, in particular thermosensitive gels like poly(N-isopropylacrylamide) (PNIPAM) that exhibit a lower critical solution temperature (LCST) at $32^{\circ} \mathrm{C}$. However, PNIPAM and other polymers exhibiting a LCST are generally less polar than PVA and can be inefficient to trap the MNPs due to the absence of hydrogen bonds and to a mesh size of typically $10-20 \mathrm{~nm}$, i.e. larger than the size of superparamagnetic iron oxide MNPs $(5-10 \mathrm{~nm}){ }^{9}$ To address this issue, several strategies were proposed as schematically represented on Figure 3, namely: a statistical copolymer network with a chelating comonomer such as 2-acetoacetoxyethylmethacrylate (AEMA), ${ }^{10}$ a semiinterpenetrated network with alginate chains wrapping the MNPs $^{11}$ or a composite network of PNIPAM and poly(ethylene glycol) (PEG) using $\mathrm{PEG}_{400}$-dimethacrylate as crosslinker of NIPAM. $^{12}$

a)

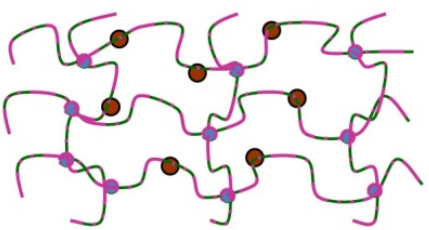

b)
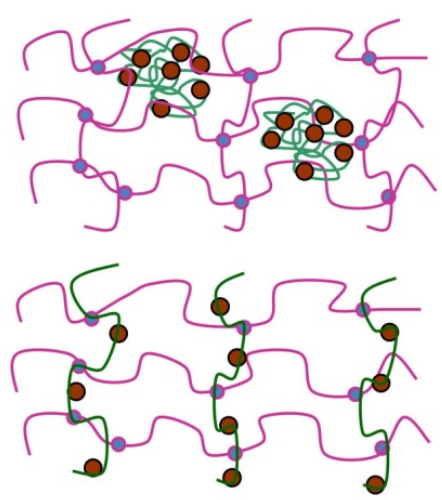

c)

Fig. 3 Three strategies proposed to trap magnetic nanoparticles, around $10 \mathrm{~nm}$ in size (brown dots), inside a hydrogel network made of a LCSTpolymer (e.g. PNIPAM, purple lines) which does not present interactions with the MNPs. Another type of chains (e.g. alginate or poly(acrylamide), green lines) strongly adsorbing onto the surface of the iron oxide is introduced either as a random copolymer (a), ${ }^{10}$ a semiinterpenetrated network (b), ${ }^{11}$ or a fully interpenetrated network (c) ${ }^{12}$

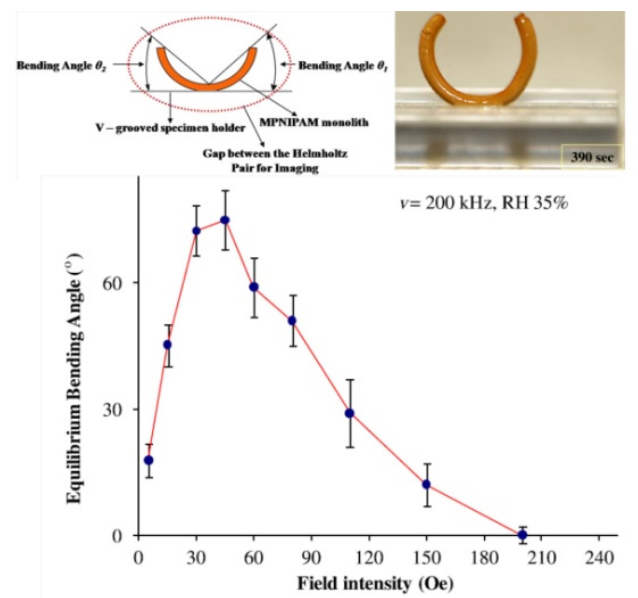

Fig. 4 Magnetic PNIPAM ferrogel cylinder lying on a grooved substrate undergoing bending under RF magnetic field due to the strain difference between the contact area with the solid under tension and the free surface under compression due to gel de-swelling when temperature reaches the LCST of PNIPAM by magnetic induction (adapted from ref. ${ }^{13}$ with permission from the IOP Publishing). 
With larger size MNPs, i.e. diameters around 25-35 nm that stand above the mesh size of the gel, a pure PNIPAM network can be used to design magnetic thermogels. ${ }^{13}$ In that case S. Gosh and T. Cai nicely evidenced an actuation mechanism based on the magnetically-induced thermal deswelling mechanism. Like a bimetal coil, a cylinder of this composite, loaded at $10 \mathrm{wt} \%$ iron oxide relatively to PNIPAM, bends at an angle that is related to the magnetic field intensity and frequency (Figure 4).

\subsection{Magneto-Active Elastomers: graduated deformations}

Magnetic polymer actuators were also developed based on a direct effect of the magnetic field through the magnetic dipolar forces acting between the magnetic fillers (at the origin of the demagnetizing and magnetostriction effects). In this case, microparticles $(1-5 \mu \mathrm{m})$ of carbonyl iron (CI, i.e. pristine $\mathrm{Fe}$, magnetically soft, made by thermal decomposition of $\left.\mathrm{Fe}(\mathrm{CO})_{5}\right)$ are dispersed in silicone-based elastomers referred as magnetorheological (MR) elastomers, ${ }^{14}$ magneto-active (MA) elastomers, $^{15}$ or magnet filler-polymer matrix composites (Magpol). ${ }^{4}$ Such materials elicit a low Young modulus $\left(E \sim 10^{6} \mathrm{~Pa}\right)$ together with a high magnetisation $\left(M>10^{4} \mathrm{~A} \cdot \mathrm{m}^{-1}\right)$ and thus exhibit large deformation, not only in field gradients but in homogeneous magnetic fields as well. The significant variation of their tensile, compression, and shear moduli with the amplitude of the applied magnetic field (a property not found for ferrogels), makes them good candidates to design active dampers for anti-vibrating applications (e.g. in automotive industry or to enhance the precision of rotating tools in mechanics workshops). Two types of MR/MA elastomers can be prepared: either isotropic (unstructured) or anisotropic (structured), the latter samples being obtained by applying a static magnetic field during the cross-linking reaction of the matrix. For magnetic silicones with a Young modulus $E=200 \mathrm{kPa}$, the maximal magneto-elastic properties were found for the pre-aligned samples, in accordance with a theoretical model based on the breaking and reorganisation of the dipolar chains at increasing strain (Figure 5 a-b). ${ }^{14}$

Experiments with a less rigid CI formulation in silicone at an even lower Young modulus, $E=16 \mathrm{kPa}$, reported that chains could still form in the soft matrix after its preparation (Figure 5 d). In that case, pre-alignment during cross-linking was not a prerequisite to obtain large magneto-elastic effects. In particular, a magnetic shape memory effect was demonstrated: such MA elastomers keep their deformation (by stretching, compression or bending) as long as a magnetic field is present, and relax elastically to their initial shape only when the field is switched off (Figure $5 \mathrm{c}-\mathrm{e}$ ). The interpretation of this magnetic shape memory effect was also based on the variation of inter-particle distances and reorganisation of dipolar chains under field. ${ }^{15}$ Other geometries of MA elastomers made of CI dispersed in silicone include magnetoelastic membranes (Figure 6) that can be used as pumps in microfluidic mixing devices propelling two fluids back-and-forth (at $10 \mathrm{~Hz}$ ) in order to accelerate their mixing. ${ }^{16}$

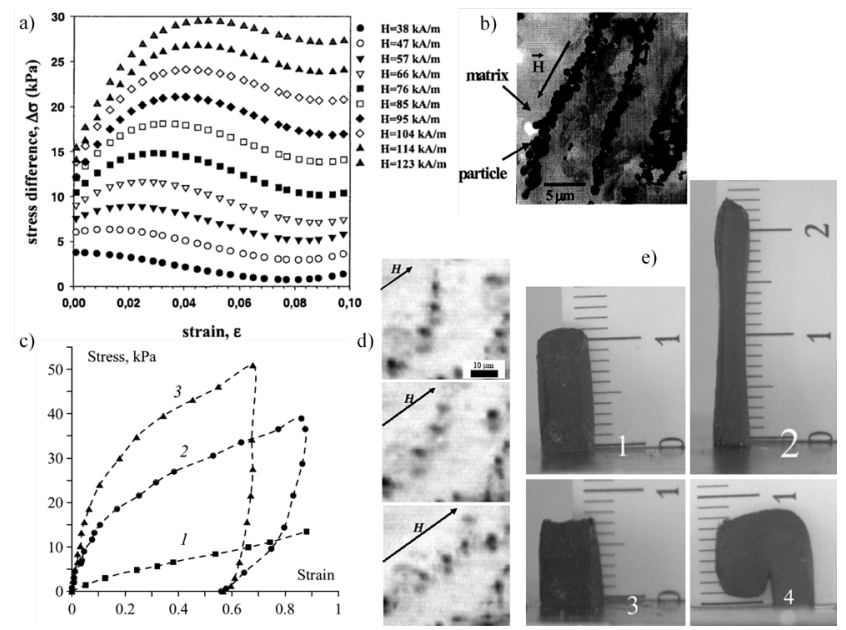

Fig. 5 Behaviours of MR/MA elastomers in homogenous magnetic fields; a-c) MR silicone loaded at $25 \mathrm{vol} \%$ with soft iron pre-aligned under a field $H=123 \mathrm{kA} / \mathrm{m}$ during the curing step; a) plot illustrating the influence of increase of magnetic field intensity $H$ on the traction curve expressed as the difference of stress $\Delta \sigma$ for a given strain $\varepsilon$ relatively to the curve without applied field $H=0$; b) optical micrograph showing chains of magnetic fillers; ${ }^{14}$; c-e) unstructured MA elastomer at $30-35 \mathrm{vol} \%$ of magnetic phase in a uniform magnetic field; c) Stress-strain dependence at (1) $H=0$, (2) $H=96 \mathrm{kA} / \mathrm{m}$, and (3) $H=208 \mathrm{kA} / \mathrm{m}$ showing a residual strain under magnetic field; d) under an increasing applied magnetic field $H$, the embedded CI fillers form chains, before returning to their initial positions when the field is switched off ; e) picture of the initial shape (1) and of the deformed shapes retained after stretching (2), contraction (3) or bending (4) as long as the magnetic field is maintained; relaxation occurs when the field is switched off (Adapted from ref. ${ }^{14}$ and ${ }^{15}$ with permission from the World Scientific Publishing Co and IOP Publishing, respectively).

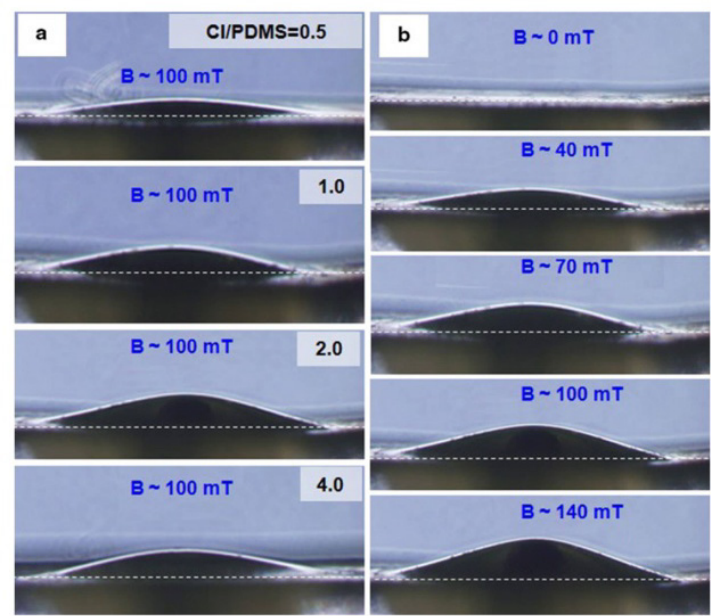

Fig. 6 a) Deflection of different membranes $(\sim 1.0 \mathrm{~mm}$ in diameter, $100 \mu \mathrm{m}$ in thickness) under the same magnetic field $(H=80 \mathrm{kA} / \mathrm{m}$, $B=100 \mathrm{mT}$ ) at increasing weight ratios CI/PDMS from 0.5 to $4 ; \mathrm{b}$ ) Deflection of the same membrane (weight ratio $=2$ ) for increasing fields up to $H=112 \mathrm{kA} / \mathrm{m}$. Such membranes can be use to design pumps or valves in a microchip (Reprinted from ref. ${ }^{16}$ with kind permission from Springer Science and Business Media).

MR/MA elastomers can also be obtained from superparamagnetic iron oxide MNPs dispersed in poly(dimethylsiloxane) (PDMS) thanks to prior functionalization of the particles surface with PDMS chains. ${ }^{17,18}$ The concept of "magnetoelastic ratio" (MER) was introduced by B. Evan et al. to quantify the propensity of a rod-like sample to bend in a 
uniform magnetic field independently of the rod length and diameter: ${ }^{17}$ it is defined as $\mu_{0} \cdot M^{2} \cdot \rho^{2} / E$ where $\mu_{0}$ is the magnetic permeability of vacuum, $M$ magnetisation, $\rho$ mass density, and $E$ the Young modulus of the composite (the deflection angle is then given by the product of the MER by the square of the rod aspect ratio). Figure 7 represents the behaviour of such magnetoelastic samples at various magnetic loads based on ferrogels $\left(E \sim 10^{3}\right.$ $\left.10^{4} \mathrm{~Pa}\right)$ or on silicone rubbers $\left(E \sim 10^{6}-10^{7} \mathrm{~Pa}\right)$. The MER enables rationalising the antagonist effects of an increase of the magnetic load in the rubber which increases magnetisation but rigidifies the elastomer.
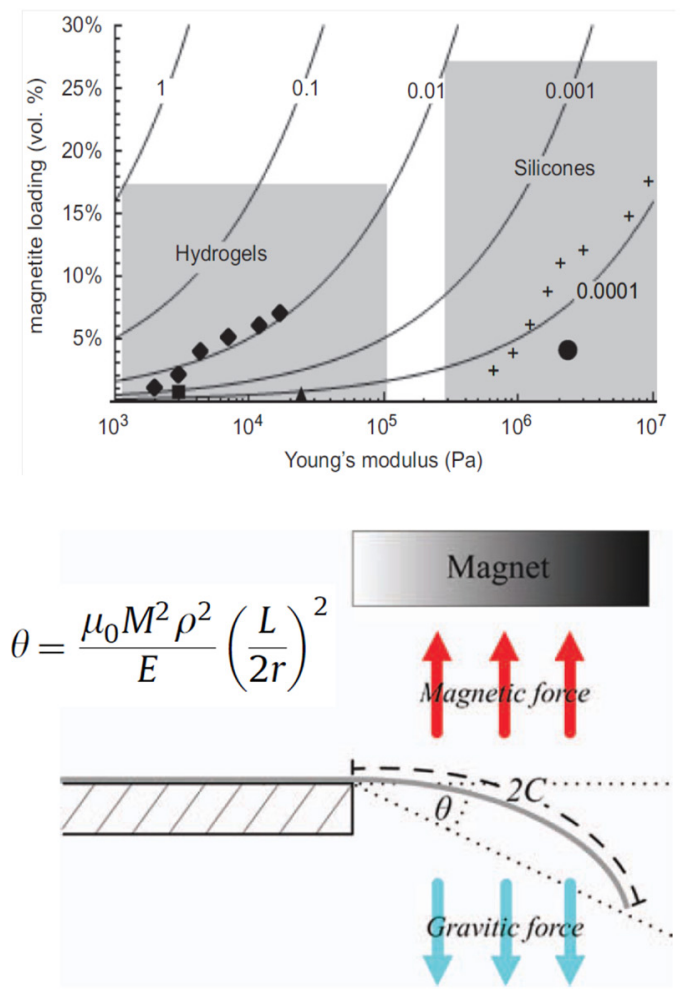

Fig. 7 Magnetoelastic ratios (MER) from literature. Solid curves represent a constant magnetoelastic ratio as a function of magnetite load and Young's modulus, e.g. $\sim 10^{-2}$ for poly(acrylamide) (PAM) ferrogels ${ }^{19}$ and $\sim 10^{-4}$ for magnetic PDMS. ${ }^{17}$ The deflection angle $\theta$ of a flexible magnetic rod is the product of MER by the square of the aspect ratio (rod length divided by its diameter) (Adapted from ref. ${ }^{17}$ and ${ }^{20}$ with permission from Elsevier and John Wiley and Sons, copyright 2012).

A last class of magnetic elastomers reported in the literature targets the tuning of optical properties with a magnetic field. Among these systems, poly(urethane) (PU) films doped at a low magnetic load, i.e. not more than $0.5 \mathrm{wt} \%$, offer an acceptable transparency in the visible spectrum combined with a magnetoelastic response. $^{21}$

\subsection{Anisotropic polymer particles for orientation control}

When preparing macroscopic magnetoelastic samples, various shapes can be obtained by the use of an appropriate mould. Practical issues can however be encountered when preparing parts of smaller dimensions (typically less than $10 \mu \mathrm{m}$ ) due to oxygen poisoning of the cross-linking/curing reaction (be it a radical polymerization for acrylate-based hydrogels or a hydrosilylation for silicones). Thus an elegant method to prepare ferrogel microparticles of various shapes (not only spherical) was introduced by $\mathrm{P}$. Doyle et $a .^{22,}{ }^{23}$. It consists in photopolymerizing droplets containing a mixture of aqueous MNPs and poly(ethylene glycol diacrylate) (PEGDA) suspended in mineral oil inside a microfluidic channel illuminated with focused UV light. The authors obtained not only magnetic ferrogel microspheres, but also microscopic disks and plugs, by squeezing the droplets in a constricting channel (with a reduced vertical dimension), ${ }^{22}$ and even Janus particles (half-magnetic), using a particular flow-focusing chip. ${ }^{23}$ Unlike macroscopic ferrogels which can deform (elongate, contract or deflect) when held by one end in a magnetic field gradient, ${ }^{24}$ the response of an assembly of microscopic ferrogels to an applied magnetic field is at first chaining. Then, when microparticles have a non isotropic symmetry, their own orientation within a chain is determined by the demagnetising effect, i.e. the particles orient their largest dimension along the field direction, as illustrated for tile-like plugs and half-spherical Janus micro-ferrogels on Figure 8.
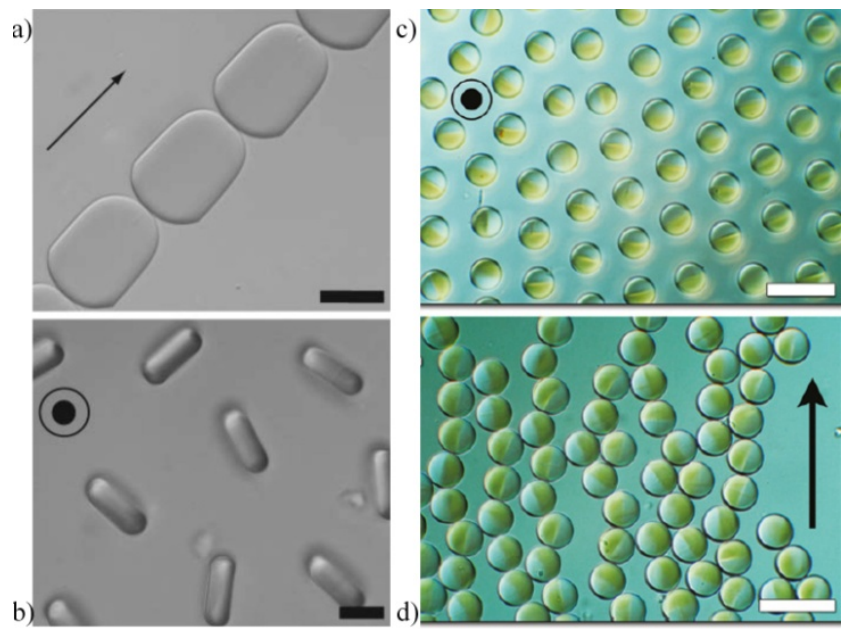

Fig. 8 Non spherical magnetic micro-hydrogels prepared by photopolymerization in microfluidics: $\operatorname{plugs}^{22}(\mathrm{a}, \mathrm{b})$ and Janus particles ${ }^{23}(\mathrm{c}, \mathrm{d})$ oriented by an in-plane (a, d) or out-of-plane magnetic field $(b, c)$. The iron oxide loads are respectively 4.9 and $3.6 \mathrm{wt} \%$ relatively to PEGDA. In both cases, the orientation is determined by the minimisation of the demagnetising field (largest dimension of magnetic body along the applied magnetic field). Scale bars are 25 (a, b) and $100 \mu \mathrm{m}$ (c, d) (Reprinted from ref. ${ }^{22}$ and ${ }^{23}$ with permission from RSC Publishing and American Chemical Society, respectively, Copyright 2013).

The microfluidic production of anisotropic magnetic microgels also enabled studying their solid rotation when submitted to a rotating magnetic field, as shown on Figure $9 \mathrm{~A}-\mathrm{H}$ for the Janus microgels of PEGDA. An analogous microfluidic approach was developed by D. Weitz et al. using doubleemulsion (MNPs in a monomer wrapped by another polymer phase and dispersed in a non miscible oil) instead of a simple one. This enabled the preparation of magnetic polystyrene (PS) cores encapsulated in a hydrophilic poly(acrylamide) (PAM) shell (Figure $9 \mathrm{a}-\mathrm{e}){ }^{25}$ The whole particle undergoes an original eccentric rotation around a point in the magnetic core under a rotating field, as shown by the trajectory of smaller particles ( $3 \mu \mathrm{m})$ surrounding the microgels. Another example deals with slightly larger PEGDA hydrogels prepared by a double emulsion process (carbonyl-iron fluid/water/silicone oil) implemented in imbricated capillaries. ${ }^{26}$ In addition to the classical translation in 
a field gradient and rotation in a rotating field (Figure 9 1-4), these core-shell microgels exhibit another original response to the application of a magnet: above a threshold field gradient, the core is torn from the shell (Figure 9, 5).
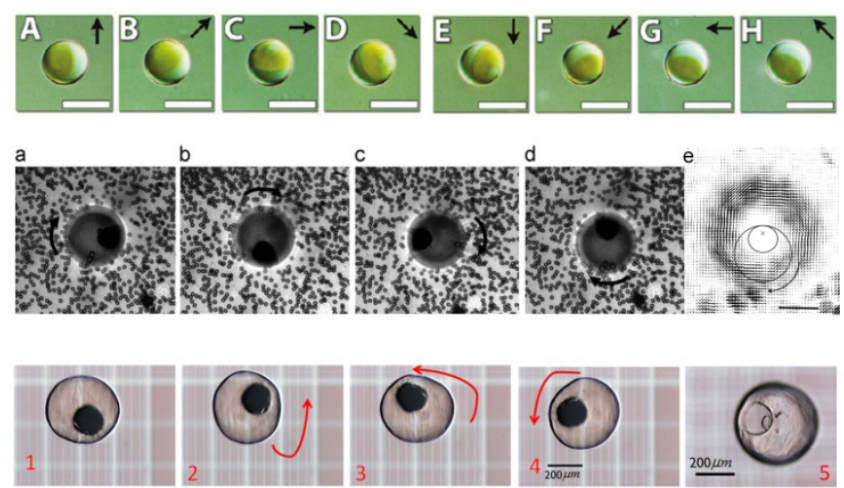

Fig. 9 Three examples of anisotropic magnetic polymer microparticles under a rotating magnetic field: (A-H) Magnetic Janus PEGDA hydrogel in a field $H=1.6 \mathrm{kA} / \mathrm{m}$ rotating in-plane $(B=2 \mathrm{mT}$, the scale bar $50 \mu \mathrm{m}) ;^{23}$ (a-d); eccentric magnetic PS core in a PAM hydrogel shell in the rotating field of a magnetic stirrer (scale bar $50 \mu \mathrm{m}$ ); e) visualisation of the flow velocity lines around the particle; ${ }^{25}(1-4) \mathrm{CI}$ core in a PEGDA hydrogel under a rotating field (scale bar $200 \mu \mathrm{m}$, adapted from ref. ${ }^{26}$ with permission from Elsevier, copyright 2013).

Other methods were reported to produce non isotropic magnetic polymer composites, in particular elongated colloids: magnetic polystyrene ellipsoids were prepared by dispersion in an elastomer membrane which is then stretched above its glass transition, ${ }^{27}$ magnetic fibres of poly(ethylene terephtalate) (PET) were electrospun; ${ }^{20}$ cylindrical magnetic needles were selfassembled by clustering of hydrophilic MNPs under a magnetic field, their electrostatic interaction with oppositely charged polymers being finely tuned by the ionic strength. ${ }^{28}$ The application of a magnetic field during the self-assembly of MNPs and polymers is a general route to produce rod-like MRPCs, for example $\mathrm{MnFe}_{2} \mathrm{O}_{4}$ and $\gamma-\mathrm{Fe}_{2} \mathrm{O}_{3}$ MNPs were co-assembled with poly(maleic anhydride-alt-1-tetradecene) using a solventdisplacement method under field. ${ }^{29}$ In these works, the usual applications proposed for such rod-like micrometric MRPCs are: magnetic stirrers for microfluidics, ${ }^{28}$ probes for rheological analysis of viscoelastic media including biological cells, ${ }^{29}$ or, less often, for "field-responsive protection" (presumably as a fabric with adjustable flexural rigidity). ${ }^{20}$

\section{Magnetically guided materials: magnetic separation and magnetic targeting}

Magnetic guidance of polymeric composites can be described as a controlled displacement of a system, composed of a magnetic material associated to a polymeric matrix, upon exposure to a magnetic field gradient. Here, the polymeric matrix may serve as a multifunctional structure able to bind or adsorb different species, allowing their later separation or simple displacement. This approach has been particularly productive in the field of biomedical applications, ranging from drug and cell delivery to diagnostic purposes. Nanosized magnetic composite materials still represent the majority of the developed systems, with a variety of morphologies and applications described. This particular subject was recently reviewed by Couvreur and colleagues. ${ }^{30}$ An overview concerning magnetic guidance of polymeric composite materials will be provided in the following paragraphs, using key examples to illustrate relevant strategies.

\subsection{Magnetic guidance for drug delivery}

It is widely accepted that the main limitations of conventional drug delivery are their difficulty to overcome the natural physiological barriers and their lack of tissue/cell specificity. In an attempt to fill this gap, one could envisage a spatial and temporal control of a cargo delivery through magnetic guidance, provided by an extracorporeal magnetic field.

The first obstacle that should be taken into account when considering magnetic guidance is the strong blood flow rate (greater than $10 \mathrm{~cm} / \mathrm{s}$ in arteries and $0.05 \mathrm{~cm} / \mathrm{s}$ in capillaries) encountered by the magnetic platforms upon intravenous administration. It is thus paramount to develop powerful magnets that can provide strong magnetic fields, able to manipulate particles against diffusion and blood stream. It is also important to mention that these inhomogeneous magnetic fields are usually effective at a distance of a few centimetres only, which can limit their final application. Indeed, and as presented in the following key examples, the majority of magnetic driven drug delivery strategies consider a fixed magnetic field that will promote a locally increased residence time, instead of a three dimensional guiding approach.

Orally administered proteins face many obstacles before being finally absorbed into the circulation, implying administration of large doses in order to achieve significant effects. A known example to illustrate this problem concerns the delivery of insulin, responsible for the regulation of blood glucose levels and that has to be intravenously administered to patients suffering from type-1 diabetes. In search of a less invasive insulin administration route, research has focused on orally administered formulations, where the hormone would be absorbed in the small intestine directly into circulation. As means to increase the delivery system residence time in the small intestine, the team of Langer developed poly(lactide-co-glycolide acid) microparticles loaded with both insulin and magnetite nanocrystals. ${ }^{31}$ Making use of the magnetic moieties of the system, the authors tested if the application of a magnetic field in the proximity of the small intestine would induce an increase of blood insulin levels, with subsequent glucose level reduction. Indeed, under magnetic exposure, the administered microparticles were able to consistently reduce glucose levels in mice, for as long as $20 \mathrm{hrs}$. Additionally, insulin levels assessment in serum confirmed this magnetically assisted insulin delivery. ${ }^{31}$

In another perspective, the use of metallic stents in the clinical practice has brought major therapeutic improvements for treatment of occlusive vascular diseases. However, in some cases a re-obstruction process, called in-stent restenosis, can occur, with associated health complications. The current clinical solution consists in the use of drug eluting stents that release potent antiproliferative drugs. Unfortunately, this approach has also led to some clinical complications. The team of Levy addressed this issue using drug-loaded nanoparticles magnetically targeted to the stent region (see Figure 10, A and B). ${ }^{32}$ The developed poly(lactic acid) nanoparticles were loaded with both magnetite nanocrystals and a non-proliferation drug 
(paclitaxel), and were shown to reduce stent restenosis, in comparison with non treated control (see Figure $10 \mathrm{C}$ and D, respectively), at 14 days post administration.
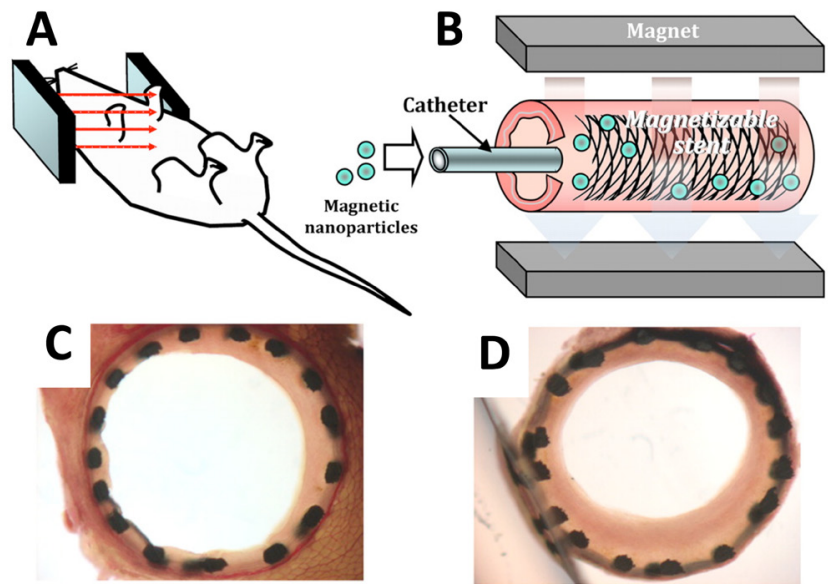

Fig.10 Targeted local delivery of magnetic nanoparticles (MNPs) to a stainless steel stent mediated by a uniform field induced magnetisation effect. The uniform field generated by paired electromagnets (A) both induces high gradients on the stent and magnetises drug-loaded nanoparticles, thus creating a magnetic force driving MNPs to the stent and adjacent arterial tissue (B). Animals treated with nanoparticles under magnetic vs. non-magnetic conditions were sacrificed, and the stented carotid segments were harvested 14 days post-surgery. As observed, upon treatment with nanoparticles under magnetic conditions a significant restenosis reduction (intra stent tissue thickness) was observed (C), in comparison to untreated controls (D). Adapted from ref. ${ }^{32}$ with permission.

In this approach a paclitaxel dose of $7.5 \mu \mathrm{g}$ per stent was used, corresponding roughly to a 4 times lower dose as compared to current drug eluting stents, ${ }^{32}$ providing a safer and more effective alternative to present methods.

In spite of great advances in cancer treatments, modern therapeutics are still facing major limitations associated with non-specific drug distribution and the inability to maintain efficient drug concentrations. These barriers have been addressed by nanotechnology, as means to control drug delivery. In this regard, the magnetically induced or assisted drug delivery can be a way to improve treatment while reducing off-target effects. Additionally, by combining magnetite nanocrystals and anticancer drugs within a polymeric platform, one can envisage the formation of a hybrid therapy and diagnostic tool (theragnostic), allowing guidance, controlled drug delivery and imaging. In an elegant study, Arias et al. demonstrated the potential of squalene-based bioconjugates, containing both magnetite nanoparticles and the anticancer drug gemcitabine, for magnetically driven cancer therapy and imaging (i.e. magnetic resonance imaging, MRI). ${ }^{33}$ Under the influence of an external magnetic field, the intravenously administered nanoparticles were capable of increasing drug accumulation in tumour tissue with subsequent tumour growth inhibition (see Figure 11, C), while allowing $\mathrm{MR}$ imaging $\left(\mathrm{T}_{2}\right.$ weighted contrast, Figure 11, A and B). ${ }^{33}$ This example emphasizes the interest of combining magnetic responsive materials with imaging and drug delivery, widening the toolbox for cancer treatment and diagnostics.

Gene therapy relies on the use of genes or sequences of nucleic acids to produce or modulate protein expression in target cells. In this case the genetic material can be faced as a pro-drug, acting at the level of cellular gene expression, as means to normalize or optimize protein expression profiles. In the case of lung pathologies the administration of drugs in the form of aerosols is widely implemented, since it constitutes a minimally invasive approach towards drug administration. With that in mind Rudolph et al. associated superparamagnetic iron oxide nanoparticles, coated with $25 \mathrm{kDa}$ poly(ethyleneimine), to plasmid DNA, in order to produce a gene therapy system for lung diseases. ${ }^{34}$ The developed formulation was nebulised during inspiration by means of intratracheal intubation in mouse lungs, while a magnet was centred above one of the lungs. The authors demonstrated that, by action of the magnetic field, a significant increase of the particle deposition occurred, associated to a decrease of particle exhalation. Additionally, a preferential accumulation was observed in the lung where the magnetic field was applied (2.5 fold increase observed for intact animals). ${ }^{34}$ Although drug efficacy was not evaluated in this study, this example broadens the use of magnetic guidance for drug delivery, taking us one step closer to real world clinical applications.

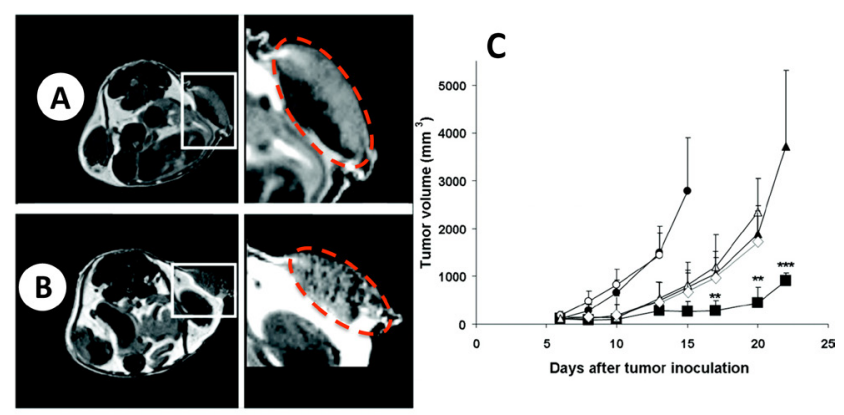

Fig.11 Examples of $\mathrm{T}_{2}$-weighted magnetic resonance images of tumours obtained at $2 \mathrm{~h}$-post injection of nanoparticles in the absence of external magnetic field (A) and the same nanoparticles guided by an external magnetic field $(1.1 \mathrm{~T})(\mathrm{B})$; in the latter case, nanoparticles were found dispersed all over the tumour tissue, as observed by the darkening of the tissue. In vivo anticancer activity of nanoparticles (with extracorporeal $1.1 \mathrm{~T}$ magnetic field) comparing the following groups: Untreated $(\bullet)$, placebo nanoparticles without drug but with magnetite $(\mathrm{O})$, free drug $(\diamond)$, nanoparticles without magnetite moieties but with $\operatorname{drug}(\boldsymbol{\Delta})$, drug/magnetite loaded nanoparticles with no extracorporeal magnetic field $(\Delta)$, drug/magnetite loaded nanoparticles with $1.1 \mathrm{~T}$ extracorporeal magnetic field (ם), in L1210 subcutaneous tumour bearing mice (C). Adapted from ref. ${ }^{33}$ with permission. Copyright 2013, American Chemical Society.

\subsection{Magnetic cell delivery/manipulation}

Cell therapy has for long been regarded as a forefront therapeutic approach, holding promise for a wide range of human pathologies. However, the control of cell localization, upon administration in the body, is a major limitation to reach clinical efficacy. In this regard, several groups have handled this question by associating magnetic material to individual cells that, upon injection, would permit cell accumulation at the targeting site by the use of a localised magnetic field. Levy and colleagues have elegantly demonstrated the magnetic guidance of endothelial cells bearing internalised magnetic nanoparticles. ${ }^{35}$ The magnetic platform consisted in albumin stabilised poly(lactide) nanoparticles containing iron oxide nanocrystals. Bovine aortic endothelial cells were incubated with the described nanoparticles 
and injected into the left ventricular cavity of rats bearing a stent in the carotid artery. The main objective was to induce stent re-endothelization, a major unsolved problem in stent angioplasty procedures. Indeed, complete and uniform cell coverage was observed after 5 minutes, when a uniform magnetic field (100mT $\approx 80 \mathrm{kA} / \mathrm{m}$ ) was applied. Conversely, in the absence of magnetic field, no magnetic nanoparticle loaded-cells where detected. The authors went further and showed that upon cell injection through a stented rat carotid, and using the same magnetic field as before, cells accumulated in the stent area only. Again, when no magnetic field was applied, no signal was observed. ${ }^{35}$
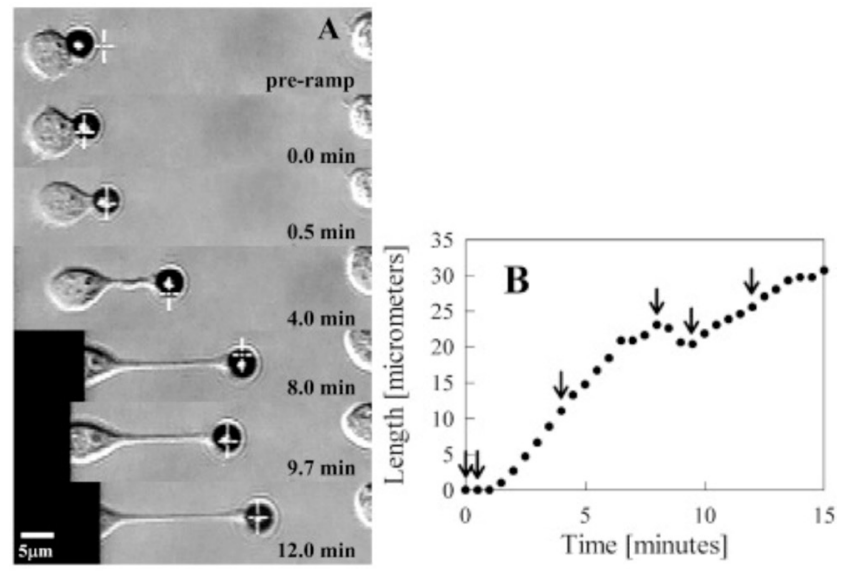

Fig.12 Neurite initiation and elongation in response to an applied force (450 pN) (A). Neurite length history corresponding to sequence in A. Arrows indicate time points corresponding to the images in A that were taken during force application (B). Adapted from ref. ${ }^{36}$ with permission from Elsevier, copyright 2013.

Upon injury to the central nervous system, unrecoverable consequences, including loss of functionality, may occur. It is fundamental to gain knowledge regarding strategies that can potentiate neural regeneration. As an example, neurite growth is of significant importance in order to produce wired functional neuronal networks. In this regard, Fass et al. focused their attention on the development of magnetic driven tools that could provide guided neurite formation. ${ }^{36}$ By the use of commercially available superparamagnetic poly(styrene) microbeads coated with anti-mouse antibodies (Dynabeads, Invitrogen), the authors demonstrated the ability to induce neurite growth in neuron cells using an external magnet. The application of a constant force $(450 \mathrm{pN})$ to the cell-attached bead, led to the formation and consequential neurite process elongation (Figure 12). Here the attachment to cells was achieved by coating the beads with an antibody that targeted a specific cell membrane protein. ${ }^{36}$ This approach allows the application of forces to individual cells with great accuracy and precision, in a minimally invasive fashion. Fundamental studies considering the mechanism of neurite formation could be addressed.

The previously exposed examples strengthen the potential of magnetic guidance towards cell delivery and manipulation. Supported by their versatility and biocompatibility, magnetic based materials may find use in future tissue engineering applications, where three-dimensional guidance and manipulation may support new technologic achievements.

\subsection{Bio/Chemo-separation applications}

The separation or concentration of certain species in solution, by means of magnetic forces, opens original perspectives towards biomedical and environmental applications. The envisaged strategies can be applied to simple molecules or to large structures like cells, using different degrees of complexity. Again, this approach has been particularly prolific concerning biomedical applications. Nevertheless, other examples, like effluent treatment, could be found and will be briefly exposed in the following paragraphs.

Protein separation by means of magnetic field may provide a simple solution for sample concentration and purification, before downstream applications (e.g. diagnostic, production). Maintaining the native protein conformation is paramount for most applications, making mild separation methods, like magnetic separation, the best choices for protein processing. Indeed, several commercial solutions are already available for magnetic protein separation, ranging from nano to micron size. In most cases the particle surface area limits the amount of protein adsorption, reducing separation efficiency. The fabrication of porous matrices has been used to circumvent this limitation. However, the use of porous materials implies slower protein diffusion rates that may increase the time for purification. With that in mind, the team of Bruening developed silica-coated magnetite nanoparticles bearing poly(2-hydroxyethyl methacrylate) brushes on their surface. ${ }^{37}$ The polymer brushes where then derivatised in order to acquire the ability to bind poly(histidine) tagged proteins. Poly(histidine) sequences are usually introduced by molecular biology techniques to provide recombinant proteins with a simple motif for subsequent purification procedures. The brush conformation allowed an increase of the surface area, without compromising diffusion rates or time of procedure, translated in superior amounts of protein purified, higher than commercially available magnetic separation systems. ${ }^{37}$

Microfluidics present unique features that make this technology best suited for the development of novel analytical systems. They allow unprecedented reduction in terms of material needed to provide measurable results, holding promise for faster and cheaper diagnostic tools. In the absence of applied forces the transport of molecules through a microfluidic device is mainly dependent on diffusion, what implies a direct limitation in terms of process efficiency. As means to address this problematic the team of Stayton and colleagues designed a microfluidic approach where small magnetic nanoparticles were used to transfer components from one flow stream to another, by means of applied magnetic field. ${ }^{38}$ Magnetic nanoparticles consisted in biotinylated poly(methacrylate-co-N-isopropylacrylamide) coated magnetic cores $\left(5 \mathrm{~nm}, \mathrm{Fe}(\mathrm{CO})_{5}\right)$. The polymeric fraction is $\mathrm{pH}$ responsive, allowing the magnetic nanoparticles to aggregate at $\mathrm{pH} 7.3$ and readily redisperse at $\mathrm{pH}$ 8.4. Biotin moieties were used in this study due to their high affinity to streptavidin. The authors mixed the magnetic nanoparticles with a fluorescently labelled streptavidin and applied it to the microfluidic system (Figure 13, A). As previously described, at $\mathrm{pH} 7.3$ the complexes aggregated in the left flow stream, therefore increasing their magnetic moment and allowing their sorting to the second (right) flow stream by means of a magnetic 
field. Once in the second stream, the complexes completely redisperse thanks to the $\mathrm{pH}$ change, allowing their high efficient recovery (ca. 80\%, Figure 13, B).
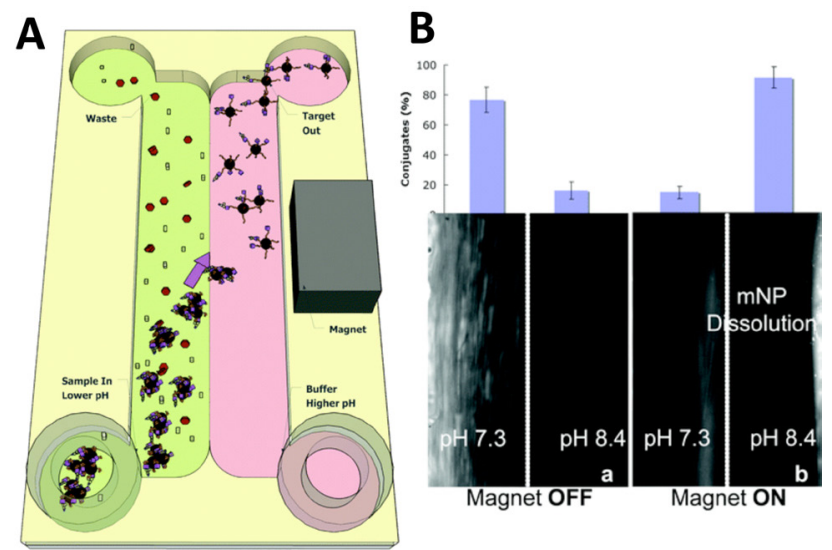

Fig.13 Target analyte separation, in a microfluidic channel, facilitated by $\mathrm{pH}$-responsive magnetic nanoparticles (MNPs). The left stream (green) is the sample that has been pre-incubated with MNPs. MNP aggregation is induced by using a lower $\mathrm{pH}$ buffer in this sample flow stream. The $\mathrm{pH}$ of the right stream (pink) is chosen to reverse MNP aggregation. A rare-earth magnet provides sufficient magnetic field to attract the aggregates laterally into the higher $\mathrm{pH}$ flow stream. The aggregates move out of the sample flow stream in the higher $\mathrm{pH}$ stream, where they return to a dispersed state, carrying the bound target analyte with them (A). Fluorescence microscopy results showing continuous stream purification of streptavidin-MNP conjugates. When the magnetic field is applied, the conjugate aggregates moved laterally across to the higher $\mathrm{pH}$ flow stream, where they redisperse. When the magnetic field is applied, most conjugates were moved into the high $\mathrm{pH}$ stream and $80 \%$ of the conjugates were collected from the right outlet (B). Adapted from ref. ${ }^{38}$ with permission of The Royal Society of Chemistry.

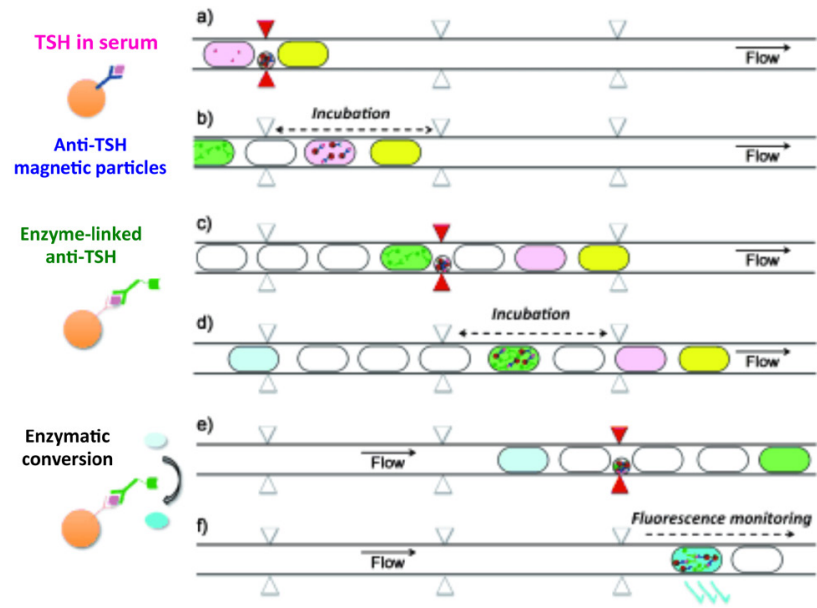

Fig.14 Diagram illustrating the basic steps involved in the magnetic immunoassay. Using an initial trap (ie. magnetic tips, opposed triangles on the scheme), antibody-grafted magnetic nanoparticles contained in the first drop were captured and transferred to a second sample drop composed of thyroid-stimulating hormone (TSH) in serum $(a, b)$. After incubation in the capillary loop following the first tweezers, the magnetic nanoparticles with the antibody complexes were trapped again in the same tweezers and washed with a droplet of buffer to remove non-specifically adsorbed proteins (c). The beads were resuspended and incubated in a fourth droplet containing the enzyme-labelled secondary antibody (d). Washing was performed in the second magnetic trap with three successive buffer droplets (e) to remove unbound secondary antibodies. Finally, the particles were released and incubated with a fluorogenic enzymatic substrate and the output was read (f). Adapted from ref. ${ }^{39}$ with permission from John Wiley and Sons, copyright 2012.
Droplet microfluidics is another interesting approach towards optimized diagnostic tools. By using antibody grafted magnetic nanoparticles, the group of Malaquin and colleagues recently developed a novel device for high-throughput nanoliter assays. ${ }^{39}$ The approach lies on the formation of nanoliter drops that travel immersed, under flow, in an oil phase inside a microtube. Using a pair of soft magnetic tips, named tweezers, the suspended antibody-grafted nanoparticles can be merged with a serumcontaining drop and used to isolate a specific content of the mixture (in this case the thyroid-stimulating hormone (TSH), Figure 14). After several steps of washing and conjugation with other antibodies, the final TSH concentration could be determined with high sensitivity. ${ }^{39}$ When compared with other standard methods (e.g. ELISA) this approach allows similar sensitivity while reducing operation time (from $2.5 \mathrm{hrs}$ to 10 min) and sample volume by 1000 times.

Biomolecules sorting by means of magnetic field may enable unprecedented reduction of analyte volume and concentration, while diminishing very significantly operation times. This has very obvious implications at the level of diagnostic costs, a major limitation in current medicine. It is expected that magnetic based technology may find use in a wide range of high sensitive and high-throughput applications.

Cell separation, by the action of external magnetic field, has for long been regarded as a forefront approach in the biomedical field. Indeed, several commercial products are available for pre-clinical research (e.g. DynaBeads ${ }^{\circledR}$, Invitrogen; MACS ${ }^{\circledR}$, Miltenyi Biotec) and diagnostics (Veridex ${ }^{\circledR}$; Johnson \& Johnson, Estapor ${ }^{\circledR}$, Merck, Adembeads, Ademtech). These approaches are based on the association of antibody grafted magnetic nanoparticles to specific cell population (by means of the antibody specificity) and to their subsequent concentration/separation by the action of an externally applied gradient magnetic field. The team of Soh took this approach one step further and envisaged the use of multitarget magnetic cell sorting, by exploring two different magnetic systems with distinctive magnetisation saturation. ${ }^{40}$ The authors used a mixture of 3 different $E$. coli bacteria - one expressing the T7 peptide, the second a streptavidin-binding peptide and a third that was not labelled - that were preincubated with two different magnetic beads. One of the beads ( $4.5 \mu \mathrm{m}$ in diameter) was labelled with an anti-T7 monoclonal antibody and the second $(2.8 \mu \mathrm{m}$ in diameter) was grafted with streptavidin. The sample was then loaded in a continuous-flow microfluidic device able to separate labelled bacteria according to their magnetisation. Indeed, this process allowed the separation of the three populations with a good purity $(>90 \%)$ in just one single pass, as determined by flow cytometry. ${ }^{40}$ Although a promising approach, the application of such a system to mammalian cells still faces some barriers. Larger structures imply a strong influence on the flow drag, making efficient cell sorting impossible with current magnetic particles. It is then imperative to develop magnetic tags with higher saturation magnetisation - ie. higher magnetic payloads - that could provide enough force for efficient cell separation. Indeed, most magnetic beads developped at this point have an iron oxide content around $50 \mathrm{wt} \%$ ( $\sim 10 \mathrm{vol} \%$, ), corresponding to Msat $\sim 30 \mathrm{kA} / \mathrm{m}$ at most.

Finally, in the ecotoxicology field, the selective treatment of 
industrial and domestic effluents is of uttermost importance and a main issue for sustained societies. Wastewaters may contain distinct doses and types of contaminants, have inherent $\mathrm{pH}$ and ionic strength properties and may include solid residues as well. It is then important to find versatile approaches, able to keep the separation process cost effective. In this sense, the development of a system that could extract contaminant, then be selectively and rapidly separated from the effluent and able to be recycled for multiple uses is of major interest. Therefore, Bee et al. associated the adsorption capacity of activated carbon with magnetic iron oxide nanoparticles (maghemite, $\gamma-\mathrm{Fe}_{2} \mathrm{O}_{3}$ ) by immersing them in an alginate matrix, forming $2.8 \mathrm{~mm}$ diameter composite beads. ${ }^{41}$ Model pollutants were used in order to test the system efficacy: positively charged methylene blue and the negatively charged methyl orange. The authors showed that the developed system was able to rapidly adsorb the proposed pollutants, independently of the $\mathrm{pH}$, and to potentially serve for a water treatment protocol. ${ }^{41}$ The applicability of such a system using real effluents has still to be tested, but this approach holds promise for the improvement of industrial and domestic effluent treatment.

\section{Magnetically actuated thermoresponsive materials: controlled drug delivery and shape shifting}

An interesting property of magnetic materials is their ability to produce heat when exposed to an alternating magnetic field (AMF). Already widely used for macroscopic magnetic devices, in for example induction cooking, this phenomenon, called inductive heating, can also be applied to polymer composites containing nanometric magnetic fillers. Indeed, when exposed to an AMF, magnetic nanoparticles generate heat through hysteresis losses (ferromagnetic particles) or through oscillation of their magnetic moment due to Néel and Brownian relaxations (superparamagnetic particles). Hence, this section will focus on temperature responsive polymer composites where the temperature variation is obtained by inductive heating, generated by the exposure to high frequency AMF. Applications where the temperature rise (hyperthermia) is the sole final response are excluded from the scope of the review since the polymer material plays no role in it.

One of the major advantages of magnetic induction is the possibility to have a non-contact remote control of temperature changes. Heat generated by the magnetic nanoparticles under exposure to AMF will trigger a phase or conformational transition of the polymer that will ultimately lead to the programmed response. This has been implemented mainly in two fields: drug delivery and shape memory materials.

It is worth noting that single domain MNPs, which have intrinsic superparamagnetic properties (above their blocking temperature, $T_{\mathrm{B}}$ ), have been largely preferred in all the applications presented here. Contrary to ferromagnetic particles, they elicit no dipolar attraction in the absence of external magnetic field. Consequently they have a better colloidal stability that favours their storage, stability upon injection or homogeneous dispersion within a polymer matrix.

\subsection{Controlled drug release}

A majority of the drug delivery systems (DDS) proposed so far elicits a release profile with an initial fast release followed by a slow continuous release. This could be a serious limitation for their application in vivo since the initial high concentration may constitute a toxicological risk and the slow release can result in under dosing, especially when considering chemotherapy. In other cases, for example when degradable particles are used, a constant release can be observed. However it is more difficult to design systems that will exclusively release their content on demand. It is therefore paramount to improve the temporal and spatial control of drug delivery.

Inductive heating can be used as a stimulus by promoting structural changes in a thermosensitive material, accompanied by the concomitant release of entrapped molecules. This strategy has been applied to design on-demand controlled drug delivery systems, mainly for cancer therapy in what has been called "magneto-chemotherapy". ${ }^{42}$ Indeed, magnetic field actuation is a very attractive mechanism for potential clinical use, mainly due to its improved tissue penetration as compared to light or heat flow. Two types of systems have been developed: particulate DDS (nano-and microparticles) for parenteral administration and macroscopic materials (mostly membranes) for implantable devices.

\subsubsection{Nano- and microparticles}

Nano- and microparticulate composites have been the most studied systems for drug delivery applications, with a wide range of structures and drug release mechanisms being proposed. Here we present some key examples illustrating various strategies that have been envisioned so far.

A first strategy consists in covering the MNPs surface with a thin polymer shell that will entrap the drug, as presented by Louguet et al. among others. ${ }^{43}$ In this work, the authors chose to use lanthanum strontium manganese oxide (LSMO) MNPs coated with a silica shell. Indeed, although iron oxide MNPs are the most widely used (mainly due to their good biocompatibility), some safety concerns regarding their in vivo application can arose due to potential tissue heating above the necrosis threshold temperature $\left(45^{\circ} \mathrm{C}\right)$, even though the actual temperature reached may be limited by thermal dissipation. This motivated the study of other types of MNPs such as LSMO, whose Curie temperature (around $50^{\circ} \mathrm{C}$ ) is well below the one of iron oxide $\left(\sim 500^{\circ} \mathrm{C}\right)$ and can be tuned by the composition of $\mathrm{La}$ and $\mathrm{Sr}$ in manganite, even though the latter exhibits inherent toxicity. In Louguet et al. work, the polymer shell was deposited through electrostatic interactions between the silica surface and hydrophilic block copolymers containing a short poly(lysine) block that covers the particle's surface. The other block is a poly(ether) segment, that forms a polymer brush, providing colloidal stability (and stealth properties), thermoresponsiveness (LCST) and a reservoir for drug loading (Figure 15). To control the thermoresponsive properties of the assemblies, the ratio of ethylene oxide and propylene oxide units could be varied in order to modify the apparent LCST of the polymer brush. Drug release was associated with the polymer brush shrinkage when a temperature above LCST is reached. As shown by the authors, this could be controlled either by raising the environment temperature or by applying an AMF. 


\section{Chem Soc Rev}

\section{Cite this: DOI: $10.1039 / \mathrm{c} 3 \mathrm{cs} 60058 \mathrm{k}$}

\section{www.rsc.org/csr}

\section{TUTORIAL REVIEW}

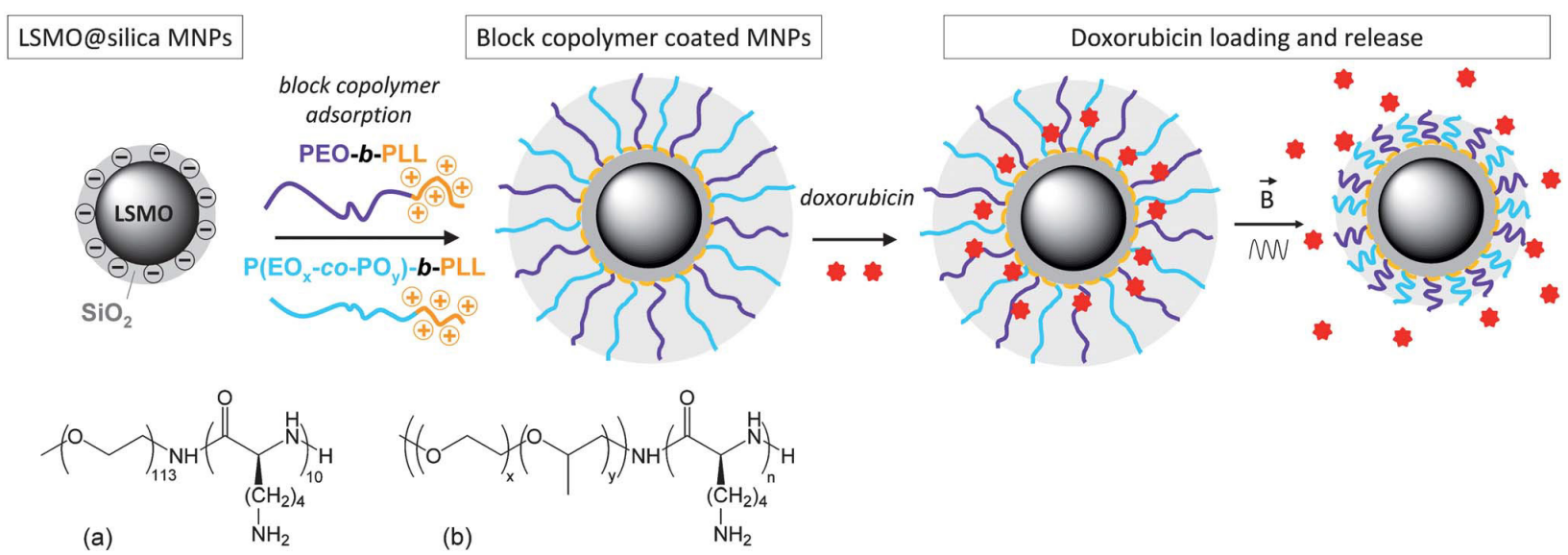

Fig.15 Preparation of magnetic responsive drug delivery systems (DDS) based on lanthanum strontium manganese oxide (LSMO) MNPs and polymer brush with a tunable LCST (top left). Chemical structures of the block copolymers used: (a), poly(ethylene glycol)- $b$-poly(lysine), and (b), poly(ethylene glycol-copropylene glycol)- $b$-poly(lysine), with $\mathrm{x}=6$ and $\mathrm{y}=29$. Principle of drug loading and alternating magnetic field (AMF) triggered drug release (right). Reprinted from ref. ${ }^{43}$ with permission of The Royal Society of Chemistry.

a)

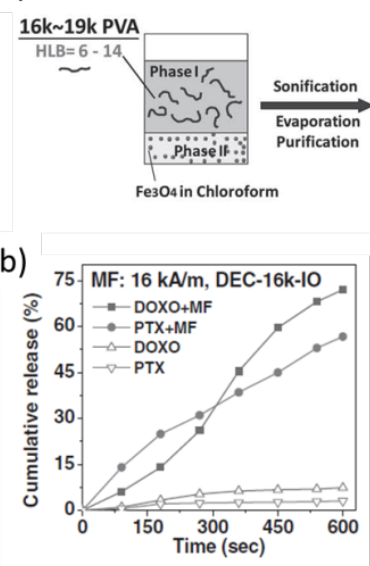

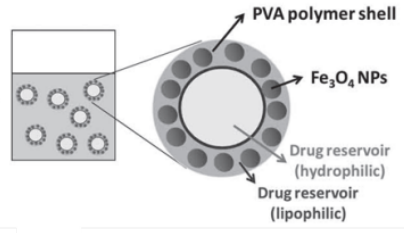

c)

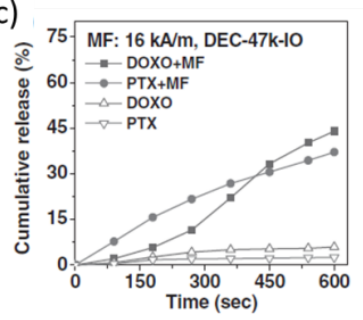

d)

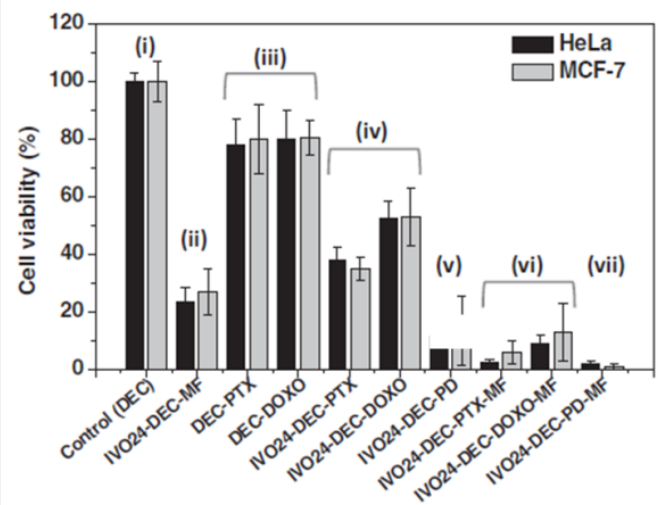

Fig.16 Schematic of one-step emulsion process for the preparation of PVA/iron oxide capsules with a PVA molecular weight of 16000 or 19000 $\mathrm{g} / \mathrm{mol}$ (a). Cumulative drug release of paclitaxel (PTX) and doxorubicin

(DOXO) with or without application of the alternating magnetic field (MF, $16 \mathrm{kA} / \mathrm{m}$ ) from capsules (DEC) prepared with PVA $16000 \mathrm{~g} / \mathrm{mol}$ (b) and 47 $000 \mathrm{~g} / \mathrm{mol}(\mathrm{c})$, showing the influence of the polymer molecular weight on the drug release rate. Cell viability after incubation for $24 \mathrm{~h}$ with capsules loaded with PTX or DOXO or both (PD), with or without MF $(16 \mathrm{kA} / \mathrm{m})$, targeted (with a cancer targeting peptide, IVO24) or not (d). Experiments show the synergistic effect of chemotherapeutics and application of the AMF. Adapted from ref. ${ }^{44}$ with permission from John Wiley and Sons, copyright 2013.

Recently, DDS presenting a capsule morphology have drawn attention thank to their ability to carry both hydrophobic and hydrophilic payloads in their aqueous core and membrane, respectively. In a recent work, the team of Prof. San-Yuan Chen developed a one-step double emulsion method for the production of nanocapsules whose membrane is constituted from poly(vinyl alcohol) and magnetite $\left(\mathrm{Fe}_{3} \mathrm{O}_{4}\right)$ nanoparticles (Figure 16, a). ${ }^{44}$ Both the preparation process (double emulsion) and the capsule morphology allow exceptionally high payloads of hydrophilic and hydrophobic drugs. This was demonstrated with two anticancer drugs, doxorubicin and paclitaxel, with loading contents up to 60 and $95 \mathrm{wt} \%$, respectively. Exposure to $\mathrm{AMF}$ drastically enhanced drug release in a way that can be tuned by the polymer molecular weight (Figure 16, b and c). The validity of such an approach was proved both in vitro and in vivo with enhanced cytotoxicity, or respective tumour volume shrinkage, when AMF was applied. Moreover, the authors showed a synergistic effect when a combination of the two anticancer agents, AMF and targeting was used (Figure 16, d).

In another study the same group prepared nanocapsules, based on poly(styrene)- $b$-poly(allyl alcohol), containing MNPs and capable of encapsulating DNA as well as hydrophobic molecules. ${ }^{45}$ Interestingly, it was shown that the quantity of DNA released under AMF $(50 \mathrm{kHz})$ was field-strength-dependent. Up to $1.2 \mathrm{kA} / \mathrm{m}$, the thermally accelerated release appeared to be a 
reversible process with no release observed when the AMF was stopped, accounting for a transitory permeabilisation of the polymer shell. But at $2.0 \mathrm{kA} / \mathrm{m}$, DNA continued to release even when the field was switched off, suggesting a permanent degradation of the polymer shell.

Also using a double emulsion method, Chiang et al. prepared poly(D,L-lactic-co-glycolic acid) (PLGA) microcapsules (1-3 $\mu \mathrm{m}$ diameter) with a $250 \mathrm{~nm}$ thick membrane containing a high density of iron oxide nanoparticles (up to $54 \mathrm{wt} \%$ ). ${ }^{46}$ The aqueous cavity of these microspheres was loaded with doxorubicin, to be used as cancer treatment via intratumoral injection. The authors demonstrated that for MNPs loading of at least $25 \mathrm{wt} \%$, the application of an AMF $(100 \mathrm{kHz}, 2.5 \mathrm{kA} / \mathrm{m})$ could induce heating above the $T_{\mathrm{g}}$ of PLGA (i.e. $40^{\circ} \mathrm{C}$ ). This allowed for the remote controlled pulsatile release of the drug, as showed on Figure 17. This finely tuned, non-contact and on-demand release of doxorubicin can allow an unprecedented control over drug concentration, adjusting the duration or number of pulses according to a patient's need or to stay in the therapeutic window.

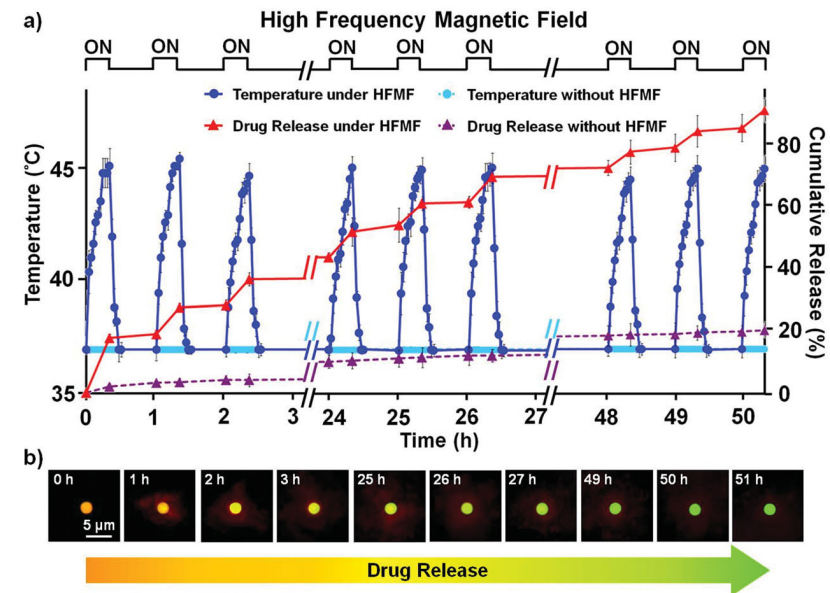

Fig.17 Doxorubicin release profiles from the iron oxide/PLGA microcapsules with and without application of high frequency magnetic field (HFMF) pulses (a). Fluorescence images showing a green-labelled microcapsule colour change as doxorubicin is released in the medium (b). Reprinted from ref. ${ }^{46}$ with permission from John Wiley and Sons, copyright 2013.

Another type of DDS presenting a capsule morphology is polymer vesicles, or polymersomes, that are particularly interesting due to their biomimetic structure (bilayered membrane). In a theragnostic approach, our group has developed polymersomes loaded with both maghemite $\left(\gamma-\mathrm{Fe}_{2} \mathrm{O}_{3}\right)$ nanoparticles and doxorubicin for combined controlled drug delivery and magnetic resonance imaging (MRI) contrast enhancement. ${ }^{42}$ When an alternating magnetic field $(500 \mathrm{kHz}$, $H=2.12 \mathrm{kA} / \mathrm{m}$ ) was applied to these particles, the drug release kinetics was significantly increased although no macroscopic heating was measured in the dispersion. It was hypothesized that a local heating at the vicinity of the MNPs leads to a permeation of the thin polymersomes membrane that increased the drug diffusion rate, ending in a faster drug release. An enhanced cell death was recently demonstrated using these "hybrid" polymersomes in vitro. ${ }^{47}$ Polymersomes loaded with $6 \mathrm{wt} \%$ doxorubicin and $30 \mathrm{wt} \%$ MNPs, internalised in HeLa cells, elicited an $18 \%$ increase in cell toxicity when a high frequency alternating magnetic field $(750 \mathrm{kHz}, H=11.2 \mathrm{kA} / \mathrm{m})$ was applied as compared to the control without magnetic field exposure.

\subsubsection{Implantable devices}

Implantable drug delivery devices are another type of DDS that can be modified for remote control by magnetic fields. For instance, composites of PNIPAM and magnetite nanoparticles have been studied by Satakar and Hilt for pulsatile drug release. ${ }^{48}$ They prepared relatively thick (1500 $\mu \mathrm{m}$ for a diameter of 15 $\mathrm{mm})$ disks in order to reduce surface to volume ratio $(\mathrm{S} / \mathrm{V})$, i.e. reduce heat transfer to the surrounding medium through diffusion, optimizing the maximum temperature that can be reached inside the material. Vitamin B12, used as a model drug, released from the composite following a Fickian profile in the absence of AMF. Its release was largely accelerated when an AMF was applied $(297 \mathrm{kHz}, 5.3 \mathrm{kA} / \mathrm{m})$. Indeed, the heat generated under AMF led the gel to de-swell and collapse (above LCST), causing the expulsion of water with a concomitant enhancement of the drug release, also favoured by the increased drug diffusivity at higher temperature. Short AMF pulses were used to obtain on-demand stepwise burst releases. Nevertheless the number of bursts was limited due to a quick exhaustion of the drug payload or to the complete collapse of the polymer matrix, which impaired further drug release.
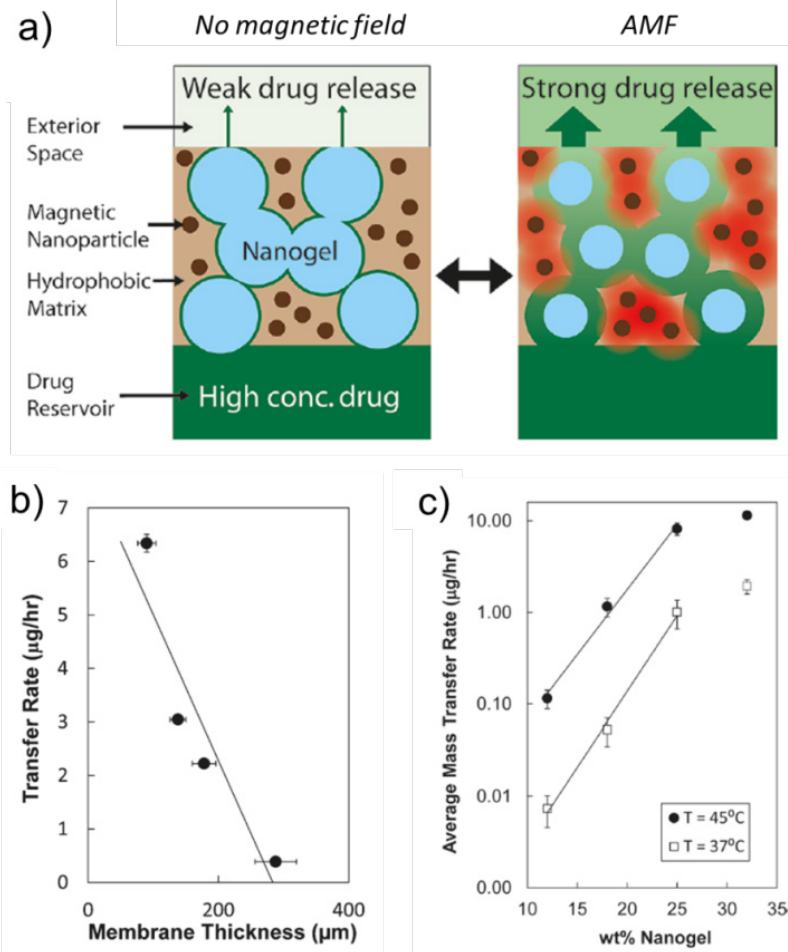

Fig.18 Schematic representation of the composite membrane valve and its functioning principle: under application of an alternating magnetic field (AMF), the magnetic nanoparticles release heat that reversibly shrinks the thermosensitive nanogels, enabling release of a drug from a reservoir (a). Rate of mass transfer of a model drug (fluorescein) as a function of membrane thickness (b) and nanogels loading (c). Adapted from ref. ${ }^{49}$ with permission. Copyright 2011, American Chemical Society.

A way to obtain sustained release over extended periods of time and/or increase the number of repetitions is to use this type of composite materials as a valve covering an implantable drug 
reservoir that will give access to a large quantity of drug. This approach was described by Hoare et al. who deposited a composite membrane, based on ethylcellulose and containing MNPs and thermoresponsive nanogels (eliciting a LCST) over a drug reservoir. ${ }^{49}$ This construction is schematically represented on Figure 18, a. Upon application of an AMF, the MNPs heat the nanogels, leading to their shrinkage and creating pores that dramatically enhance the drug release rate. This release rate, as well as the on-off drug release ratio, could be tuned by the membrane thickness (Figure 18, b) and the nanogel loading (Figure 18, c). As expected, the frequency and power of the applied AMF also affects the drug dosing by changing the steady state temperature of the device. This tunability opens new avenues in the development of adaptable therapeutic tools, providing unprecedented control over real time drug dosing.

\subsection{Shape memory materials}

Shape memory polymers are capable of performing important shape changes, after application of a programming process usually a deformation at fixed temperature - in order to recover their original shape. Generally they are thermoplastic reticulated materials with a glass and/or melting transition temperature. After deformation at a temperature higher than their transition temperature, the temporary shape is fixed by rapid cooling (Figure 19, a). Then, the original shape can be recovered by heating again above the transition temperature (Figure 19, a and b). Such materials can be useful for applications in a wide range of fields such as defence, smart textiles, packaging, aerospace, adaptive optics, robotics and biomedical engineering, like magnetic actuators (see part 1) or minimal invasive surgery devices.

(a)
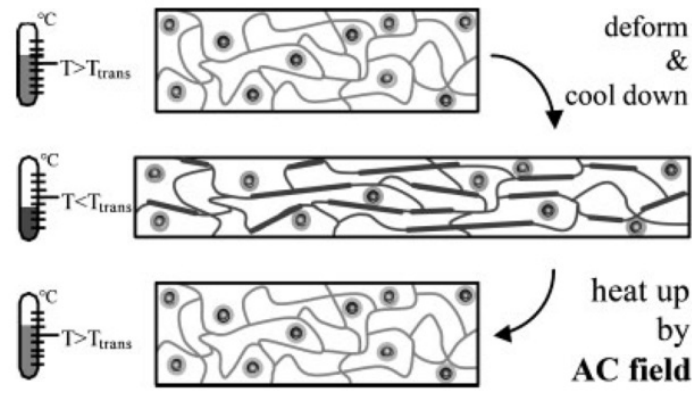

(b)

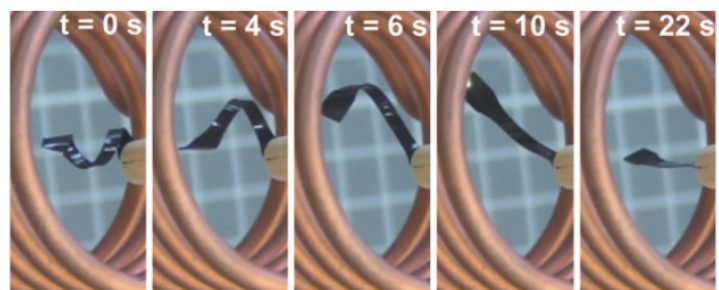

Fig.19 Schematic representation of the alternating magnetic field (AMF) induced shape memory effect in shape-memory polymers composites (a). The permanent shape (top) is transformed in a second, temporary shape by a programming process (deformation at $T>T_{\text {trans }}$ ). The temporary shape is stabilised by the crystalline phase of the polymer, represented as rigid segments (middle). Induction heating leads to a temperature increase inside the matrix, above the transition temperature $\left(T_{\text {trans }}\right)$, that induces the permanent shape recovery (bottom). Series of pictures illustrating the previously described AMF $(258 \mathrm{kHz}, H=30 \mathrm{kA} / \mathrm{m})$ induced shape recovery process for a poly(ether urethane) composite $(10 \mathrm{wt} \%$ magnetic nanoparticles) sample that was deformed into a spiral (b). Adapted from ref. ${ }^{50}$ and ${ }^{51}$ with permission.

Thermoplastic shape-memory polymers (SMP) have been adapted for magnetic remote activation by filling them with MNPs. The thermally induced shape shifting of such materials can then be triggered by exposing them to an alternating magnetic field. Indeed, the heat generated by the MNPs, under alternating magnetic field, can trigger a useful phase transitions of SMPs.

Early works concerned materials with dual-shape properties, i.e. one thermal transition at a temperature $T_{\text {trans }}$ that was either a melting temperature $\left(T_{\mathrm{m}}\right)^{50,51}$ or a glass transition $\left(T_{\mathrm{g}}\right) .^{51}$ To prepare these composites, relatively high amounts of MNPs (at least $10 \mathrm{wt} \%$ ) were dispersed in the monomer mixture before polymerization. To favour the preparation of homogeneous materials (evenly distributed filler), Mohr et al. used silica coated iron oxide NPs in order to reduce agglomeration into $\mu \mathrm{m}$-sized clusters that could sediment during the polymerization process. ${ }^{51}$

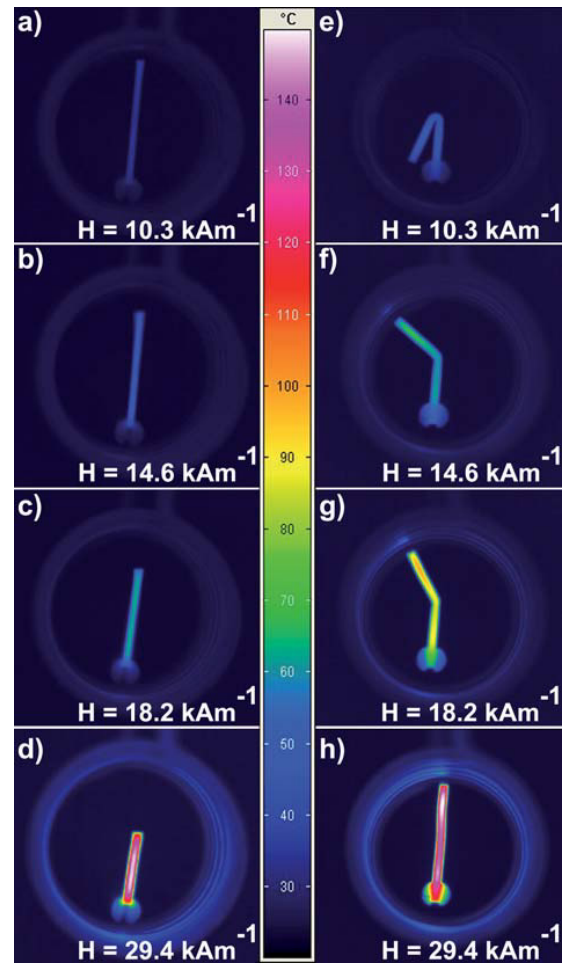

Fig.20 Pictures taken with an infrared camera of the shape recovery at different magnetic field strengths of two samples made of the same composite but deformed with two different methods. Sample on the left side (a-d) was deformed by elongation whereas sample on the right side (e-h) underwent a bending process. The temperature reached inside the material appears to depend on the programming process, with a recovery at higher field strength for the sample deformed by elongation. This highlights the influence of the surface to volume ratio $(\mathrm{S} / \mathrm{V})$ in the design of shape memory polymer composite systems. Reprinted from ref. ${ }^{52}$ with permission of The Royal Society of Chemistry.

In an elegant study, Lendlein group prepared triple-shape polymer composites - two thermal transitions-, containing poly $(\varepsilon-$ caprolactone) (PCL) and poly(cyclohexyl methacrylate) (PCHMA) segments, for non-contact actuation. ${ }^{52}$ They showed that MNPs incorporation had little influence on the thermal properties of the polymer but impacted on the mechanical properties, significantly decreasing the elongation at break. 
Hence, the filler content should be a compromise between the heating properties (higher reachable temperature) and the mechanical properties. Another important parameter that should be taken into account in order to gain a good control over shape recovery is the surface to volume ratio $(\mathrm{S} / \mathrm{V})$. As observed in Figure 20, depending on $\mathrm{S} / \mathrm{V}$ ratio, the heat loss (by exchanges with the surrounding environment) can vary, what may imply large differences in terms of the magnetic field strength necessary to attain the transition temperature. Finally, excellent triple shape properties with complete recovery of the original shape were obtained for composites containing $40 \mathrm{wt} \%$ of PCL. A step-wise increase of the magnetic field strength allowed the recovery of the intermediate and original shapes in a sequential manner.

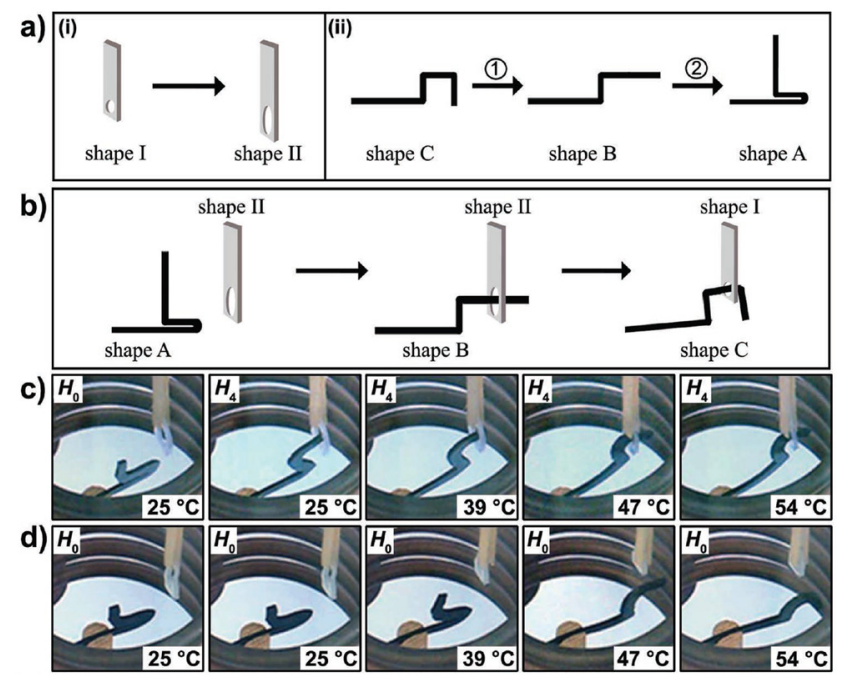

Fig.21 Principle of the fixation device or the "active hook" experiment: shape programming of the two parts (a) and working principle of the fixation (b). Images taken at different environmental temperatures: environmental heating at $H_{4}=20.2 \mathrm{kA} / \mathrm{m}$ (c) and environmental heating without magnetic field $(H=0, \mathrm{~d})$. Reprinted from ref. ${ }^{53}$ with permission from John Wiley and Sons, copyright 2013.

More recently, the same group showed that it is possible to adjust the apparent switching temperature of a shape memory composite by combining contributions from two sources: the heat energy coming from the magnetic field actuation and the heat flow from the environment. ${ }^{53}$ Experimental measures revealed that environmental and inductive heating contribute additively to the temperature in the composite material, the latter being the environment temperature plus the contribution of the inductive heating. In a simple approach, the contribution of the inductive heating could be expressed as $\Delta T_{\mathrm{mag}}=k \cdot H^{2}$ where $H$ is the magnetic field strength and $k$ is a material related constant. Consequently, under AMF, the apparent - environmental switching temperature of the shape memory polymer can be decreased. To demonstrate this principle experimentally, the authors built a fixation device consisting of thermoresponsive hook and lock (Figure 21). To successfully activate the device, the hook shall enter the fixation hole before this part changes shape. As shown in Figure 21, this was achieved by filling the hook with MNPs and by applying the appropriate AMF. This work demonstrated that by combining different heat sources it is possible to obtain a complex response from a thermosensitive composite system.
Lendlein and collaborators also showed that it was possible to prepare magnetic memory composites by filling temperaturememory polymers with MNPs. ${ }^{54}$ Temperature memory polymers can remember the temperature where they were deformed. This effect results from the fact that they have broad transitions and that the mechanical deformation is fixed by the volume fraction of the domains associated with this transition $\left(T_{\mathrm{m}}\right.$ or $\left.T_{\mathrm{g}}\right)$. Since for a given composite material, the temperature reached in the material corresponds to a given magnetic field strength, the temperature memory corresponds to a magnetic memory. This property was illustrated in an experiment where two identical samples, of the same composite material, were deformed at different field strength. Each sample recovers its original shape only when the field at which it was deformed is reached.

The previous examples of shape-programming systems and drug release triggered by $a c$ magnetic fields illustrate the variety and complexity of devices that can be thought of in future drug delivery and robotic applications, the ever increasing control over the fine tuning of the magnetic actuation opening new perspectives for high precision applications.

\section{Conclusions}

To date, composites of polymer and magnetic nanoparticles are the most elegant and efficient way to obtain magnetic responsive polymer materials exhibiting high amplitude magneto-response. These MRPCs can be divided in three classes with regards to their intended applications and/or mechanisms involved in the response to the magnetic field. A first group gathers ferrogels, microsized hydrogels and other elastomeric materials that can be deformed in a controlled manner in homogeneous fields or gradiends. Another class that we identified is composed of polymers designed for interactions with biomolecules or biostructures (biocompatible, bioresponsive polymers). In this field, MRPCs were successfully employed for magnetic guidance of drug delivery systems, manipulation of individual cells and separation in complex biological media or environmental samples. Finally, MRPCs prepared from thermoresponsive polymers can be remotely activated using alternating magnetic fields. This strategy was especially used for controlled drug delivery with injectable or implantable devices and in the shapememory polymers area.

In order to perfectly anticipate the properties of these MRPCs, many parameters need to be controlled (Table 1). Especially, the geometrical parameters of the devices (size, shape, surface/volume ratio, aspect ratio) and the nanostructure/composition of the composite (ie. magnetic material loading, compatibility between the polymer and magnetic phases) are relevant parameters that drive the amplitude of the desired magneto-response. Therefore, a careful multiscale design is necessary to fully adjust the final properties. In particular, reducing the sample size can have a positive impact on the response kinetics. However, when considering heat exchanges, the decreased diffusion time, associated with an increased surface area (diffusion time is scaling like the square of the sample characteristic size), can also be a drawback, limiting the maximal temperature that should be reached by magnetic induction with $a c$ fields. Another important point is the adjustment of polymers transition temperatures to just a few 
degrees above the body temperature. Finally, the obtention of very soft materials (e.g. low mechanical moduli) is also requested, a practical thumb-rule being the calculation of the MER factor described in Section 1.

Magnetic remote control is particularly interesting and has already demonstrated promising results for biomedical applications. Indeed, even if the concept of MRPCs has initially been established for material sciences and actuators, it is now impacting significantly the biomedical field. Most living systems are not sensitive to magnetic field, rendering this actuation strategy specific and potentially harmless. In addition, the possibility to have long distance effects, using a magnetic field gradient or alternating magnetic field, opens new avenues for realistic remote actuation of therapeutic or diagnosis devices. In this sense, nanosized magnetic responsive composite materials present several characteristics making them optimal for therapeutic applications. Due to their small size, one can envisage their intravenous or localized administration, in a minimally invasive fashion. Additionally, and owing to the versatility of the polymeric structure the association with other components (e.g. drugs, tracing agents) can be easily achieved. The magnetic moieties will then enable the spatial manipulation and permit to trigger a relevant therapeutic effect (e.g. drug release, hyperthermia) by means of the application of an external magnetic field. Moreover, due to the inherent nanoparticle high surface area and ease of manipulation, by means of magnetic gradients, such MRCP nanoparticles may allow in a near future to revolutionize the fields of molecular manipulation and separation at the nanoscale.

Knowledge of the physical mechanisms associated with the polymer materials response to magnetic actuation is of uppermost importance for bio-engineers involved in the inception of such new technologies, especially for devices implemented in the body or drug delivery vehicles embarked in the systemic circulation. One can therefore anticipate future promising developments of MRPCs and their implementation in devices that will particularly impact the biomedical field.

Table 1 Main parameters for the control of MRPC properties depending of the intended application.

\begin{tabular}{|c|c|c|c|}
\hline & Magneto-elastic deformations & Magnetic guidance, Magnetic separation & $\begin{array}{l}\text { AMF-induced thermal phase/shape } \\
\text { transition }\end{array}$ \\
\hline $\begin{array}{l}\text { Magnetic } \\
\text { fillers }\end{array}$ & $\begin{array}{l}\text { Controlled sizes: } \\
\rightarrow \text { Ferrogels: magnetic core size or } \\
\text { clusters size superior to gel mesh-size } \\
\rightarrow \text { Silicones: micrometric size inducing } \\
\text { particle chaining when submitted to } \\
\quad d c \text { magnetic field }\end{array}$ & $\begin{array}{l}\text { - High loading content to reach high } \\
\text { magnetisation } \\
\text { - Biocompatible magnetic materials } \\
\text { (surface passivation required when MNPs } \\
\text { are not made of pure iron oxide to shield } \\
\text { from the toxicity of "hard" nanomagnets) }\end{array}$ & $\begin{array}{l}\text { - Homogeneous distribution in the } \\
\text { polymer matrix } \\
\text { - High loading content } \\
\text { - High specific absorption rate (SAR) to } \\
\text { maximise the heating source }\end{array}$ \\
\hline $\begin{array}{l}\text { Polymer } \\
\text { matrix }\end{array}$ & $\begin{array}{l}\text { - Material as soft as possible to optimize } \\
\text { the magneto-elastic ratio (balance of } \\
\text { magnetisation vs. mechanical strength) }\end{array}$ & $\begin{array}{l}\text { - Small MRPC particles size to increase } \\
\text { half-life in blood circulation and } \\
\text { minimize drag force } \\
\text { - Controlled of surface properties to } \\
\text { finely tune the interactions with } \\
\text { biostructures depending on the } \\
\text { application } \\
\text { - High porosity for environmental } \\
\text { purposes (remediation) }\end{array}$ & $\begin{array}{l}\rightarrow \text { Drug Release: } \\
\text { - Finely tuned } T_{\text {trans }} \text { to meet the } \\
\text { biological constraints }\left(40-45^{\circ} \mathrm{C}\right) \\
\text { - Control of the physico-chemical } \\
\text { mechanisms leading to drug release: } \\
\text { permeability vs. degradation } \\
\rightarrow \text { Shape memory devices: } \\
\text { - minimize the } \mathrm{S} / \mathrm{V} \text { ratio } \\
\text { For both: controlled heat dissipation } \\
\text { (size, heat conductivities) }\end{array}$ \\
\hline
\end{tabular}

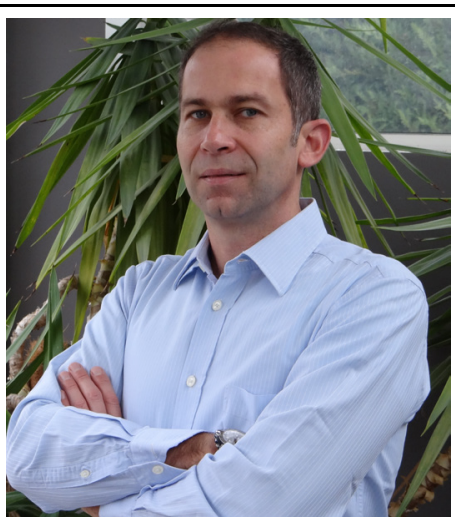

Sébastien Lecommandoux is Full Professor at the University of Bordeaux (ENSCBP-IPB) and is leading the group "Polymer Nanotechnology and Life Sciences" at the Laboratoire de Chimie des Polymères Organiques (LCPO-CNRS). His current research interests include polypeptide and polysaccharide based block copolymers self-assembly, biomimetic approaches toward design of synthetic viruses and cells as well as drug-delivery. He is deputy director of the LCPO and director of the research at the IPB-ENSCBP. He is also currently chairing the ESF Research Network Programme on "Precision Polymer materials" $\mathrm{P}^{2} \mathrm{M}$. Sébastien Lecommandoux is recipient of the CNRS bronze medal award (2004) and is honorarium junior member of the Institut Universitaire de France (promotion IUF 2007). $\mathrm{He}$ is Associate Editor for
Biomacromolecules (ACS) and in the Editorial Advisory Board of several international journals, including Polymer Chemistry and Biomaterials Science (RSC).

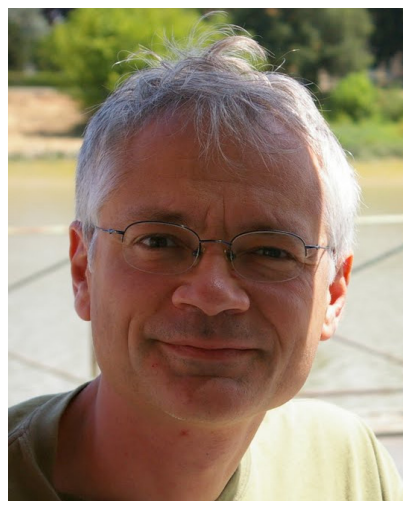

Olivier Sandre is tenured CNRS researcher since 2001. He joined the Laboratory of Organic Polymers Chemistry in 2010 after collaborating with LCPO since 2004. He works on polymeric systems doped with magnetic nanoparticles, especially magnetic polymersomes for theranostics (MRI combined with anticancer therapy). He was nominated adjunct professor of Waterloo University in Canada and received the 2012 Young Researcher award of the Physical Chemistry division of French Chemical Society (SCF) and French Physics Society (SFP) for his researches on selfassembled magnetic polymer composite materials. 


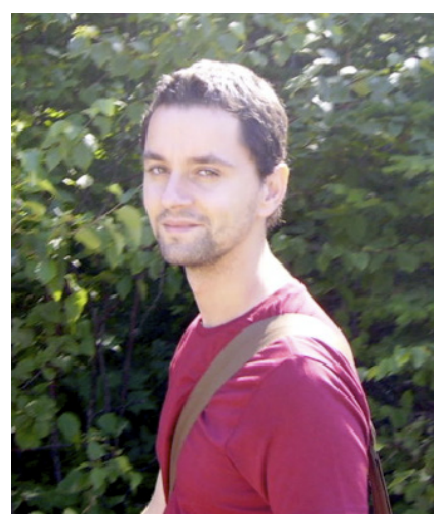

Hugo Oliveira is a postdoctoral fellow in Sébastien Lecommandoux's group, Laboratoire de Chimie des Polymères Organiques (LCPO-CNRS). Here he participated on the European project Nanother that focused on the development of novel nanoparticle systems that permit controlled drug release and imaging (MRI), while targeting specific cancer tissues. He obtained his $\mathrm{PhD}$ in Biomedical Engineering in 2010 from the University of Porto and the Instituto de Engenharia Biomédica (INEB), Portugal. In 2012 he received the Young Scientist award at the $9^{\text {th }}$ World Biomaterials Congress in Chengdu, China.

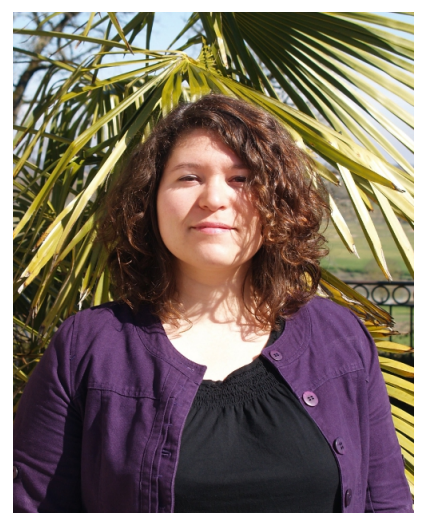

Julie Thevenot is a postdoctoral fellow in Sébastien Lecommandoux's group in Laboratoire de Chimie des Polymères Organiques (LCPO). She obtained her $\mathrm{PhD}$ from University of Lyon, France, in 2007 working on polymer/lipid nanoconstructs for nucleic acid and protein delivery. Her research interests include polymer chemistry, polymerbased drug delivery systems, physical chemistry of colloidal suspensions and self-assembly. At LCPO, she contributed to the European project Nanother that focused on the development of novel hybrid (polymer/magnetic inorganic material) nanoparticle systems for cancer therapy and diagnosis.

\section{Notes and references}

${ }^{a}$ Université de Bordeaux, LCPO, UMR 5629, F-33600 Pessac, France. CNRS, LCPO, UMR 5629, F-33600 Pessac, France. Fax: (+) 3354000 84 87; E-mail: lecommandoux@enscbp.fr; osandre@enscbp.fr * Corresponding author

Financial support from the European Commission under the seventh framework within the frame of the NanoTher project (CP-IP 213631-2), ESF RNP "Precision Polymer Materials" P2M, CNRS-MI G3N ("NanoBlast"), and COST actions TD1004 and 1007 is acknowledged.

1. M. A. Cohen Stuart, W. T. S. Huck, J. Genzer, M. Muller, C. Ober, M. Stamm, G. B. Sukhorukov, I. Szleifer, V. V. Tsukruk, M. Urban, F. Winnik, S. Zauscher, I. Luzinov and S. Minko, Nat Mater, 2010, 9, 101-113.

2. P.-G. De Gennes, M. Hébert and R. Kant, Macromolecular Symposia, 1997, 113, 39-49.

3. M. H. Li, P. Keller, J. Yang and P. A. Albouy, Advanced Materials, 2004, 16, 1922-1925.

4. V. Q. Nguyen, A. S. Ahmed and R. V. Ramanujan, Advanced Materials, 2012, 24, 4041-4054
5. C. S. Brazel, Pharmaceutical Research, 2009, 26, 644-656.

6. Q. Dai and A. Nelson, Chemical Society Reviews, 2010, 39, 4057 4066.

7. S. F. Medeiros, A. M. Santos, H. Fessi and A. Elaïssari, International Journal of Pharmaceutics, 2011, 403, 139-161.

8. M. Zrinyi, L. Barsi and A. Buki, The Journal of Chemical Physics, 1996, 104, 8750-8756.

9. J. A. Galicia, F. Cousin, E. Dubois, O. Sandre, V. Cabuil and R. Perzynski, Soft Matter, 2009, 5, 2614-2624.

10. P. C. Papaphilippou, A. Pourgouris, O. Marinica, A. Taculescu, G. I. Athanasopoulos, L. Vekas and T. Krasia-Christoforou, Journal of Magnetism and Magnetic Materials, 2011, 323, 557-563.

11. Z. Li, J. Shen, H. Ma, X. Lu, M. Shi, N. Li and M. Ye, Polymer Bulletin, 2012, 68, 1153-1169.

12. N. S. Satarkar and J. Zach Hilt, Acta Biomaterialia, 2007, 4, 11-16.

13. S. Ghosh and T. Cai, Journal of Physics D: Applied Physics, 2010, 43.

14. C. Bellan and G. Bossis, International Journal of Modern Physics B, 2002, 16, 2447-2453.

15. G. V. Stepanov, D. Y. Borin, L. R. Yu, P. V. Melenev and N. S. Perov, Journal of Physics: Condensed Matter, 2008, 20, 204121.

16. J. Li, M. Zhang, L. Wang, W. Li, P. Sheng and W. Wen, Microfluidics and Nanofluidics, 2011, 10, 919-925.

17. B. A. Evans, B. L. Fiser, W. J. Prins, D. J. Rapp, A. R. Shields, D. R. Glass and R. Superfine, Journal of Magnetism and Magnetic Materials, 2012, 324, 501-507.

18. C. Sötebier, A. Michel and J. Fresnais, Applied Sciences, 2012, 2, 485-495.

19. J. A. Galicia, O. Sandre, F. Cousin, D. Guemghar, C. Menager and V. Cabuil, Journal of Physics-Condensed Matter, 2003, 15, S1379-S1402.

20. B. W. Ahn and T. J. Kang, Journal of Applied Polymer Science, 2012, 125, 1567-1575.

21. M. Yoonessi, J. A. Peck, J. L. Bail, R. B. Rogers, B. A. Lerch and M. A. Meador, ACS Applied Materials \& Interfaces, 2011, 3, 2686-2693.

22. D. K. Hwang, D. Dendukuri and P. S. Doyle, Lab on a Chip, 2008, 8, 1640-1647.

23. K. P. Yuet, D. K. Hwang, R. Haghgooie and P. S. Doyle, Langmuir, 2009, 26, 4281-4287.

24. M. Zrinyi, L. Barsi and A. Buki, Polymer Gels and Networks, 1997, 5, 415-427.

25. C.-H. Chen, A. R. Abate, D. Lee, E. M. Terentjev and D. A. Weitz, Advanced Materials, 2009, 21, 3201-3204.

26. J. Rodriguez-Lopez, H. C. Shum, L. Elvira, F. Montero de Espinosa and D. A. Weitz, Journal of Magnetism and Magnetic Materials, 2013, 326, 220-224.

27. C. Herrmann, M. B. Bannwarth, K. Landfester and D. Crespy, Macromolecular Chemistry and Physics, 2012, 213, 829-838.

28. J. Fresnais, J. F. Berret, B. Frka-Petesic, O. Sandre and R. Perzynski, Advanced Materials, 2008, 20, 3877-3881.

29. M. Allione, B. Torre, A. Casu, A. Falqui, P. Piacenza, R. Di Corato, T. Pellegrino and A. Diaspro, Journal of Applied Physics, 2011, 110. 
30. L. H. Reddy, J. L. Arias, J. Nicolas and P. Couvreur, Chem Rev, 2012, 112, 5818-5878.

31. J. Cheng, B. A. Teply, S. Y. Jeong, C. H. Yim, D. Ho, I. Sherifi, S. Jon, O. C. Farokhzad, A. Khademhosseini and R. S. Langer, Pharm Res, 2006, 23, 557-564.

32. M. Chorny, I. Fishbein, B. B. Yellen, I. S. Alferiev, M. Bakay, S. Ganta, R. Adamo, M. Amiji, G. Friedman and R. J. Levy, Proc Natl Acad Sci U S A, 2010, 107, 8346-8351.

33. J. L. Arias, L. H. Reddy, M. Othman, B. Gillet, D. Desmaele, F. Zouhiri, F. Dosio, R. Gref and P. Couvreur, ACS Nano, 2011, 5, 1513-1521.

34. P. Dames, B. Gleich, A. Flemmer, K. Hajek, N. Seidl, F. Wiekhorst, D. Eberbeck, I. Bittmann, C. Bergemann, T. Weyh, L. Trahms, J. Rosenecker and C. Rudolph, Nat Nanotechnol, 2007, 2, 495499.

35. B. Polyak, I. Fishbein, M. Chorny, I. Alferiev, D. Williams, B. Yellen, G. Friedman and R. J. Levy, Proc Natl Acad Sci U S A, 2008, 105, 698-703.

36. J. N. Fass and D. J. Odde, Biophys J, 2003, 85, 623-636.

37. F. Xu, J. H. Geiger, G. L. Baker and M. L. Bruening, Langmuir, 2011, 27, 3106-3112.

38. J. J. Lai, K. E. Nelson, M. A. Nash, A. S. Hoffman, P. Yager and P. S. Stayton, Lab Chip, 2009, 9, 1997-2002.

39. A. Ali-Cherif, S. Begolo, S. Descroix, J. L. Viovy and L. Malaquin, Angew Chem Int Ed Engl, 2012, 51, 10765-10769.

40. J. D. Adams, U. Kim and H. T. Soh, Proc Natl Acad Sci U S A, 2008, 105, 18165-18170.

41. V. Rocher, J. M. Siaugue, V. Cabuil and A. Bee, Water Res, 2008, 42, 1290-1298.

42. C. Sanson, O. Diou, J. Thévenot, E. Ibarboure, A. Soum, A. Brûlet, S. Miraux, E. Thiaudière, S. Tan, A. Brisson, V. Dupuis, O. Sandre and S. Lecommandoux, ACS Nano, 2011, 5, 11221140 .

43. S. Louguet, B. Rousseau, R. Epherre, N. Guidolin, G. Goglio, S. Mornet, E. Duguet, S. Lecommandoux and C. Schatz, Polymer Chemistry, 2012, 3, 1408-1417.

44. S.-H. Hu, B.-J. Liao, C.-S. Chiang, P.-J. Chen, I. W. Chen and S.-Y. Chen, Advanced Materials, 2012, 24, 3627-3632.

45. S.-H. Hu, S.-Y. Chen and X. Gao, ACS Nano, 2012, 6, 2558-2565.

46. W.-L. Chiang, C.-J. Ke, Z.-X. Liao, S.-Y. Chen, F.-R. Chen, C.-Y. Tsai, Y. Xia and H.-W. Sung, Small, 2012, 8, 3584-3588.

47. H. Oliveira, E. Pérez-Andrés, J. Thevenot, O. Sandre, E. Berra and S. Lecommandoux, Journal of Controlled Release, 2013, DOI: 10.1016/j.jconrel.2013.1001.1013.

48. N. S. Satarkar and J. Z. Hilt, Journal of Controlled Release, 2008, 130, 246-251.

49. T. Hoare, B. P. Timko, J. Santamaria, G. F. Goya, S. Irusta, S. Lau, C. F. Stefanescu, D. Lin, R. Langer and D. S. Kohane, Nano Letters, 2011, 11, 1395-1400.

50. A. M. Schmidt, Macromolecular Rapid Communications, 2006, 27, 1168-1172.

51. R. Mohr, K. Kratz, T. Weigel, M. Lucka-Gabor, M. Moneke and A. Lendlein, Proceedings of the National Academy of Sciences of the United States of America, 2006, 103, 3540-3545.

52. U. N. Kumar, K. Kratz, W. Wagermaier, M. Behl and A. Lendlein, Journal of Materials Chemistry, 2010, 20, 3404-3415.
53. U. N. Kumar, K. Kratz, M. Heuchel, M. Behl and A. Lendlein, Advanced Materials, 2011, 23, 4157-4162.

54. M. Y. Razzaq, M. Behl and A. Lendlein, Advanced Functional Materials, 2012, 22, 184-191. 